\title{
On a theory of the $b$-function in positive characteristic
}

\author{
Thomas Bitoun ${ }^{1}$
}

Published online: 2 February 2018

(C) The Author(s) 2018. This article is an open access publication

\begin{abstract}
We present a theory of the $b$-function (or Bernstein-Sato polynomial) in positive characteristic. Let $f$ be a non-constant polynomial with coefficients in a perfect field $k$ of characteristic $p>0$. Its $b$-function $b_{f}$ is defined to be an ideal of the algebra of continuous $k$-valued functions on $\mathbb{Z}_{p}$. The zero-locus of the $b$-function is thus naturally interpreted as a subset of $\mathbb{Z}_{p}$, which we call the set of roots of $b_{f}$. We prove that $b_{f}$ has finitely many roots and that they are negative rational numbers. Our construction builds on an earlier work of Mustaţă and is in terms of $D$-modules, where $D$ is the ring of Grothendieck differential operators. We use the Frobenius to obtain finiteness properties of $b_{f}$ and relate it to the test ideals of $f$.
\end{abstract}

Mathematics Subject Classification 13A35 - 14F10

\section{Contents}

Introduction . . . . . . . . . . . . . . . . . . . . . . . . 3502

1 Construction . . . . . . . . . . . . . . . . . . . . 3505

1.1 The algebra of higher Euler operators . . . . . . . . . . . . . . . . . . . . 3505

1.2 Definition of the $b$-function . . . . . . . . . . . . . . . . . . . . . . 3509

1.3 Bounded level versions of $N_{f} \ldots \ldots \ldots \ldots \ldots \ldots$

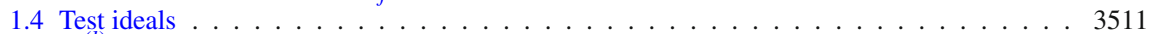

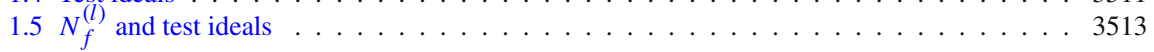

2 Relation to test ideals and rationality of the roots . . . . . . . . . . . . . . . 3516

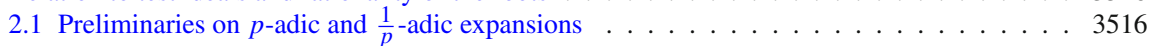

2.2 Unit $F$-modules . . . . . . . . . . . . . . . . . . . . . . . . . . . . . . . 3518

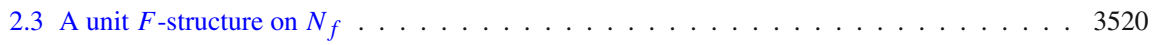

Thomas Bitoun

tbitoun@gmail.com

1 Mathematical Institute, University of Oxford, Oxford OX2 6GG, UK

Birkhäuser 
2.4 The roots of the $b$-function and $F$-jumping exponents . . . . . . . . . . . . . . . 3525

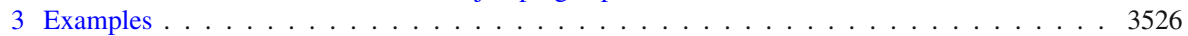

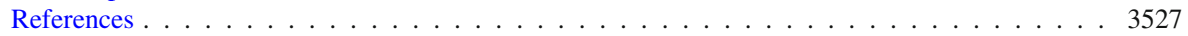

\section{Introduction}

\section{Bernstein-Sato polynomial}

Let $f$ be a non-constant complex polynomial in $n$ variables. Let us consider after Bernstein the functional equation in $P(s)$ and $q(s)$ :

$$
P(s) f^{s+1}=q(s) f^{s},
$$

where $P(s)$ is an $s$-polynomial with coefficients in differential operators with polynomial coefficients in $n$ variables and $q(s)$ is a polynomial of the variable $s$. The fundamental result is that there is always a solution $(P(s), q(s))$ of the functional equation such that the polynomial $q(s)$ is non-zero [3, Theorem 1']. Since moreover the second components $q(s)$ of the solutions obviously form an ideal of $\mathbb{C}[s]$, one may consider the monic generator $b_{f}(s)$ of that ideal. It is called the Bernstein-Sato polynomial (or the $b$-function) of $f$.

The roots of the Bernstein-Sato polynomial are not arbitrary. Indeed Kashiwara showed the following [13, Corollary (5.2)]:

Theorem A The roots of $b_{f}(s)$ are strictly negative rational numbers.

The consideration of the solutions to the functional equation above was originally motivated by the problem of analytically continuing the zeta function attached to $f$, see [3, Theorem 1]. We would like to highlight two other types of consequences of that result.

On one hand the existence of the Bernstein-Sato polynomial of a general nonconstant polynomial is of theoretical interest for modules over rings of differential operators or $\mathcal{D}$-modules. Namely it was the latter's first application and has been crucial in singling out the importance of holonomicity [3, Corollary (1.4.b)]. Moreover it is used to prove the stability of holonomicity under operations, e.g. direct image for an open embedding [4, p. 25], nearby and vanishing cycles [2, 2.1. Key Lemma].

On the other hand, the Bernstein-Sato polynomial of $f$ is a rich invariant of the singularity $\{f=0\}$, as was discovered by Malgrange [19]. In particular the roots of $b_{f}(s)$ are related to very many invariants of the singularities of $f$, see $[15,22]$ and [17] for surveys. Of motivational interest for us is the following result of EinLazarsfeld-Smith-Varolin [8, Theorem B], relating the jumping coefficients of the multiplier ideals of $f$ to the roots of $b_{f}(s)$, see [8] for the definitions:

Theorem B Let $\lambda$ be a jumping coefficient of $f$ which lies in $(0,1]$. Then $-\lambda$ is a root of the Bernstein-Sato polynomial of $f$.

In this paper, we put forth a natural definition of Bernstein-Sato polynomial (or $b$-function) in positive characteristic and show that it satisfies statements analogous to Theorems A and B. 


\section{Positive characteristic}

Let $k$ be a perfect field of positive characteristic $p$ and let $g$ be a non-constant $k$ polynomial in $n$ variables. A positive characteristic analogue of the multiplier ideals is provided by the (generalised) test ideals of Hara and Yoshida [12]. Where the $F$ jumping exponents of the test ideals of $g$ are the pendant to the jumping coefficients of the multiplier ideals. These are rational numbers [6, Theorem 3.1].

In accordance with the problematic set forth by Mustaţă in [20] and in analogy with Theorem B, we would like the sought for $b$-function of $g$ to be related to its $F$-jumping exponents. It does thus seem preferable for the $b$-function to be an object living beyond the positive characteristic.

The theory we propose here is $p$-adic, in the sense that the roots of our $b$-function are naturally $p$-adic integers. To describe it, let us first consider the usual abstraction of Bernstein's functional equation, see e.g. [13, §1].

Let $\mathcal{D}$ be the ring of complex differential operators with polynomial coefficients in $n$ variables, and let $\mathcal{O}$ be the ring of complex polynomials in $n$ variables. We use the notation $\mathcal{D}[s]$ for the algebra $\mathcal{D} \otimes \mathbb{C} \mathbb{C}[s]$, where the variable $s$ is central. For a non-constant element $f$ of $\mathcal{O}$, let $\mathcal{O}\left[\frac{1}{f}\right][s] f^{s}$ be the free $\mathcal{O}\left[\frac{1}{f}\right][s]$-module of rank one with generator $f^{s}$. It is endowed with a compatible action of $\mathcal{D}[s]$ by making a derivation $\partial$ in $\mathcal{D}$ act on the generator $f^{s}$ by $\partial \cdot f^{s}:=s \partial(f) f^{-1} f^{s}$. The left $\mathcal{D}[s]-$ module $\mathcal{D}[s] f^{s}$ is the $\mathcal{D}[s]$-submodule of $\mathcal{O}\left[\frac{1}{f}\right][s] f^{s}$ generated by $f^{s}$ and the one generated by $f \cdot f^{s}$ is denoted $\mathcal{D}[s] f^{s+1}$. It is clear that there is a differential operator $P(s) \in \mathcal{D}[s]$ such that $(P(s), q(s))$ is a solution to Bernstein's functional equation $(\star)$ if and only if the polynomial $q(s)$ annihilates the $\mathcal{D}[s]$-module $\mathcal{D}[s] f^{s} / \mathcal{D}[s] f^{s+1}$. Thus the Bernstein-Sato polynomial of $f$ is the monic generator of the annihilator of the action of $\mathbb{C}[s]$ on $\mathcal{D}[s] f^{s} / \mathcal{D}[s] f^{s+1}$.

In the positive characteristic theory, the rôle of $\mathbb{C}[s]$ is played by the $k$-algebra $A_{k}$ generated by the binomial coefficients functions modulo $p$. It is not principal but there is nevertheless a natural notion of roots of an ideal. Indeed $A_{k}$ is isomorphic to $k\left[Y_{e} ; e \in \mathbb{N}\right] /\left(Y_{e}^{p}-Y_{e} ; e \in \mathbb{N}\right)$ and the generators are canonically ordered. Thus for each homomorphism $\alpha$ from $A_{k}$ to a field, $\alpha\left(Y_{e}\right)$ is in the prime field, for all $e$. We associate to $\alpha$ the $p$-adic integer $\Sigma_{e} \alpha_{e} p^{e}$, where $\alpha_{e}$ is the unique lift of $\alpha\left(Y_{e}\right)$ to $\{0, \ldots, p-1\}$. In particular to each maximal ideal of $A_{k}$, we may attach a $p$ adic integer for $\alpha$ the canonical quotient homomorphism. In fact, we show that $A_{k}$ is isomorphic to the $k$-algebra $\Lambda_{k}$ of continuous $k$-valued functions on the $p$-adic integers. Thus to each ideal $I$ of $A_{k}$ corresponds a set of $p$-adic integers. Namely those associated by the above to the maximal ideals containing $I$. We call them the roots of $I$.

Our approach to the $b$-function of a non-constant polynomial $g$ in positive characteristic is to consider a left $D \otimes_{k} A_{k}$-module $N_{g}^{\gamma}$ for $D$ the ring of Grothendieck differential operators, which is thought of as the positive characteristic analogue of $\mathcal{D}[s] f^{s} / \mathcal{D}[s] f^{s+1}$ (for $g$ instead of $f$ ), see Definition 1.2.4. We then define the $b$ function $b_{g}$ of $g$ to be the annihilator of the action of $A_{k}$ on $N_{g}^{\gamma}$. The roots of $b_{g}$ satisfy strong finiteness properties. Indeed, here are those corresponding to Theorems A and B: 
Theorem $\mathbf{C}$ The roots of the b-function of $g$ form a finite set of strictly negative rational numbers. Furthermore they are not smaller than -1 .

In fact, we have the following precise relation to the $F$-jumping exponents of $g$, see Theorem 2.4.1.

Theorem $\mathbf{D}$ Let $\lambda$ be an $F$-jumping exponent of $g$ in $(0,1] \cap \mathbb{Z}_{(p)}$. Then $-\lambda$ is a root of the b-function of $g$. Moreover this exhausts the roots of $b_{g}$.

In order to prove our main results, we study in some depth the $D$-module structure of $N_{g}^{\gamma}$. We show that it is a finitely generated unit $F$-module (Theorem 2.3.4), which serves as a replacement for holonomicity. In fact we exhibit an explicit generator of the unit $F$-module $N_{g}^{\gamma}$ expressed in terms of the test ideals of $g$. This is what ultimately allows us to relate the roots of the $b$-function to the $F$-jumping exponents and prove Theorem 2.4.1.

\section{Further comments}

\section{Relation to the work of Mustaţă and motivation}

In [20], which was our starting point, Mustaţă uses the action of Euler operators on bounded level analogues of $N_{g}$ to construct a whole sequence of BernsteinSato-type polynomials and his main result is that the information provided by these invariants corresponds to that provided by the $F$-jumping exponents of $g$. However, this correspondence leaves open the question of what is the natural analogue of the Bernstein-Sato polynomial. This is the question addressed here. We do so by focusing on $D$-modules (as opposed to $D^{(e)}$-modules with bounded divided powers) and hence on the whole algebra of higher Euler operators. This leads us to a new notion of $b$-function (or Bernstein-Sato polynomial), naturally $p$-adic.

\section{Nearby cycles}

We have generalised our theory to unit $F$-modules coefficients and argue that these may be called nearby cycles. This will appear elsewhere.

\section{Frobenius structure}

The action of Frobenius on $N_{g}$ is given here via an explicit root for the corresponding unit $F$-structure. It would be preferable to have a direct description. We have failed to obtain one so far.

\section{Relation to the Bernstein-Sato polynomial}

In view of the analogy between the Bernstein-Sato polynomial of a, say, complex polynomial and the theory of the $b$-function presented here, it is natural to ask about the comparison between the Bernstein-Sato polynomial of a polynomial $f$ with say 
rational coefficients and the $b$-function of its reduction $f_{p}$ modulo a prime $p$, for $p$ large.

The situation is subtle, as shown in Sect. 3. Strikingly, there are polynomials $f$ such that there are arbitrary large primes $p$ for which there is a root of the $b$-function of $f_{p}$ which is not a root of the Bernstein-Sato polynomial of $f$, see Example 3.0.8.

\section{Construction}

\subsection{The algebra of higher Euler operators}

Let $k$ be a field of characteristic $p>0$ and let $R$ be a commutative $k$-algebra. In the sequel, we will use the notation $D_{R}$ for the ring of global sections of the sheaf of Grothendieck differential operators $D_{S p e c(R) / k}$. We refer to $[11,16.8]$ for a general treatment of the sheaf of rings of Grothendieck differential operators.

After [20], we set the higher Euler operators to be the following differential operators in $D_{k[t]}$ :

Definition 1.1.1 Let $e \geq 0$ be a natural number. The $e$-th higher Euler operator is

$$
v_{e}:=\frac{d}{d t}^{\left[p^{e}\right]} t^{p^{e}}
$$

where $\frac{d}{d t}^{\left[p^{e}\right]}$ is the divided power differential operator such that for all $n \geq 0$, $\frac{d}{d t}{ }^{\left[p^{e}\right]}\left(t^{n}\right)=\left(\begin{array}{c}n \\ p^{e}\end{array}\right) t^{n-p^{e}}$. (We set $\left(\begin{array}{c}n \\ m\end{array}\right)=0$ for $n<m$.)

Definition 1.1.2 The $k$-algebra of higher Euler operators $\Gamma_{k}$ is the unital $k$-subalgebra of $D_{k[t]}$ generated by the higher Euler operators.

For all $n \geq 0$, let $\left(\begin{array}{l}s \\ n\end{array}\right)$ denote the $n$-th binomial coefficient function modulo $p$,

$$
\mathbb{N} \rightarrow \mathbb{F}_{p}, l \mapsto\left(\begin{array}{l}
l \\
n
\end{array}\right)
$$

We will consider them as $k$-valued functions.

Definition 1.1.3 The k-algebra of binomial coefficients $k\left[\left(\begin{array}{c}s \\ p^{0}\end{array}\right),\left(\begin{array}{c}s \\ p^{1}\end{array}\right),\left(\begin{array}{c}s \\ p^{2}\end{array}\right), \ldots\right]$ is the $k$-subalgebra of the algebra of $k$-valued functions on $\mathbb{N}$ generated by the binomial coefficients functions $\left\{\left(\begin{array}{l}s \\ n\end{array}\right) ; n \in \mathbb{N}\right\}$.

Let us recall the classical theorem of Lucas [10].

Theorem 1.1.4 Let $m$ and $n$ be natural numbers and let $p$ be a prime number. The binomial coefficient $\left(\begin{array}{c}m \\ n\end{array}\right)$ modulo $p$ is given by:

$$
\left(\begin{array}{c}
m \\
n
\end{array}\right)=\Pi_{e=0}^{\infty}\left(\begin{array}{c}
m_{e} \\
n_{e}
\end{array}\right) \bmod p
$$


where $m=\Sigma_{e=0}^{\infty} m_{e} p^{e}$ (resp. $\left.n=\Sigma_{e=0}^{\infty} n_{e} p^{e}\right)$ is the base $p$ expansion of $m$ (resp. $n$ ). (We set $\left(\begin{array}{l}0 \\ 0\end{array}\right)=1$.)

Corollary 1.1.5 The k-algebra of binomial coefficients is generated by the binomial coefficients $\left\{\left(\begin{array}{c}s \\ p^{e}\end{array}\right) ; e \in \mathbb{N}\right\}$. This justifies our choice of notation. Moreover, the kernel of the surjective morphism of $k$-algebras $\pi_{k}$ :

$$
k\left[Y_{e} ; e \geq 0\right] \rightarrow k\left[\left(\begin{array}{c}
s \\
p^{0}
\end{array}\right),\left(\begin{array}{c}
s \\
p^{1}
\end{array}\right),\left(\begin{array}{c}
s \\
p^{2}
\end{array}\right), \ldots\right], Y_{e} \mapsto\left(\begin{array}{c}
s \\
p^{e}
\end{array}\right)
$$

is the ideal generated by $\left\{Y_{e}^{p}-Y_{e} ; e \in \mathbb{N}\right\}$.

Proof Let $n$ be a natural number and let $n=\Sigma_{e=0}^{N} n_{e} p^{e}$ be its base $p$ expansion. It follows directly from Lucas' Theorem that

$$
\left(\begin{array}{l}
s \\
n
\end{array}\right)=\Pi_{e=0}^{N}\left(\begin{array}{c}
s \\
p^{e}
\end{array}\right)
$$

This proves that the $k$-algebra of binomial coefficients is generated by the binomial coefficients $\left\{\left(\begin{array}{c}s \\ p^{e}\end{array}\right) ; e \in \mathbb{N}\right\}$.

Since the $\left(\begin{array}{c}s \\ p^{e}\end{array}\right)$ are $\mathbb{F}_{p}$-valued functions, it is clear that they satisfy the relations $\left(\begin{array}{c}s \\ p^{e}\end{array}\right)^{p}=\left(\begin{array}{c}s \\ p^{e}\end{array}\right)$. Thus $\left\{Y_{e}^{p}-Y_{e} ; e \in \mathbb{N}\right\}$ is contained in the kernel of $\pi_{k}$. Let us prove that the kernel is generated by $\left\{Y_{e}^{p}-Y_{e} ; e \in \mathbb{N}\right\}$. Since $\pi_{k}=k \otimes_{\mathbb{F}_{p}} \pi_{\mathbb{F}_{p}}$ and $k$ is flat over $\mathbb{F}_{p}$, it is enough to show the assertion for $\pi_{\mathbb{F}_{p}}$.

Let $P$ be in the kernel. That is $P$ is a $\mathbb{F}_{p}$-polynomial in $N$ variables for a natural number $N$ and $P\left(\left(\begin{array}{c}s \\ p^{0}\end{array}\right), \ldots,\left(\begin{array}{c}s \\ p^{N-1}\end{array}\right)\right)=0$. Thus by Lucas' Theorem, $P$ is in the kernel of the evaluation morphism:

$$
\mathbb{F}_{p}\left[Y_{0}, \ldots, Y_{N-1}\right] \rightarrow \operatorname{Fun}\left(\mathbb{F}_{p}^{N}, \mathbb{F}_{p}\right)
$$

where $F$ un $\left(\mathbb{F}_{p}^{N}, \mathbb{F}_{p}\right)$ is the algebra of $\mathbb{F}_{p}$-valued functions on $\mathbb{F}_{p}^{N}$. One easily sees by counting dimensions that this implies that $P$ lies in the ideal generated by $\left\{Y_{e}^{p}-Y_{e} ; 0 \leq\right.$ $e \leq N-1\}$. This concludes the proof of the corollary.

The $k$-algebra of higher Euler operators and the $k$-algebra of binomial coefficients are isomorphic. Let us fix an isomorphism.

Lemma 1.1.6 The assignment $\left(\begin{array}{c}s \\ p^{e}\end{array}\right) \mapsto-v_{e}$ induces a morphism $\gamma$ of $k$-algebras:

$$
k\left[\left(\begin{array}{c}
s \\
p^{0}
\end{array}\right),\left(\begin{array}{c}
s \\
p^{1}
\end{array}\right),\left(\begin{array}{c}
s \\
p^{2}
\end{array}\right), \ldots\right] \stackrel{\gamma}{\rightarrow} \Gamma_{k} .
$$

The morphism $\gamma$ is an isomorphism. 
Proof Let us first note that the definition of the higher Euler operators provides a natural embedding of $\Gamma_{k}$ into the algebra of binomial coefficients. Indeed, since $D_{\mathbb{A}_{k}^{1}}$ acts faithfully on $k[t]$, the higher Euler operators are characterised by their action on the monomials. It is given by $v_{e}\left(t^{n}\right)=\frac{d}{d t}{ }^{\left[p^{e}\right]} t^{p^{e}}\left(t^{n}\right)=\left(\begin{array}{c}p^{e}+n \\ p^{e}\end{array}\right) t^{n}=\left(1+\left(\begin{array}{c}n \\ p^{e}\end{array}\right)\right) t^{n}$, the last equality holding by Lucas' Theorem. We thus see that the assignment $v_{e} \mapsto 1+\left(\begin{array}{c}s \\ p^{e}\end{array}\right)$ defines an embedding of $\Gamma_{k}$ into the $k$-algebra of binomial coefficients. It clearly is an isomorphism.

We are thus left with having to show that the assignment $\left(\begin{array}{c}s \\ p^{e}\end{array}\right) \mapsto-1-\left(\begin{array}{c}s \\ p^{e}\end{array}\right)$ induces an automorphism of $k\left[\left(\begin{array}{c}s \\ p^{0}\end{array}\right),\left(\begin{array}{c}s \\ p^{1}\end{array}\right),\left(\begin{array}{c}s \\ p^{2}\end{array}\right), \ldots\right]$. This is obvious from the presentation given in Corollary 1.1.5.

Remark 1.1.7 The isomorphism $\gamma$ is the analogue of the isomorphism

$$
\mathbb{C}[s] \rightarrow \mathbb{C}\left[\frac{d}{d t} t\right], \quad s \mapsto-\frac{d}{d t} t
$$

from the complex theory.

Next we explicitly relate the algebra of binomial coefficients to the $p$-adic integers.

Theorem 1.1.8 Let $\mathbb{F}_{p}$ and $k$ be endowed with the discrete topology.

(1) For all natural numbers $e$, the binomial coefficient function $\left(\begin{array}{c}s \\ p^{e}\end{array}\right)$ modulo p extends to a continuous $\mathbb{F}_{p}$-valued function $c_{e}$ on $\mathbb{Z}_{p}$, such that $c_{e}(z)=z_{e} \bmod p$, where $z=\Sigma_{e \geq 0 z_{e}} p^{e}$ is the $p$-adic expansion. This induces an embedding $i$ of k-algebras:

$$
k\left[\left(\begin{array}{c}
s \\
p^{0}
\end{array}\right),\left(\begin{array}{c}
s \\
p^{1}
\end{array}\right),\left(\begin{array}{c}
s \\
p^{2}
\end{array}\right), \ldots\right] \stackrel{i}{\rightarrow} C\left(\mathbb{Z}_{p}, k\right),
$$

where $C\left(\mathbb{Z}_{p}, k\right)$ is the $k$-algebra of continuous $k$-valued functions on $\mathbb{Z}_{p}$. The morphism $i$ is an isomorphism.

(2) Moreover, for each p-adic integer $z$, let $e v_{z}$ be the evaluation morphism: $C\left(\mathbb{Z}_{p}, k\right) \rightarrow k, f \mapsto f(z)$. The map $\epsilon$ from the $p$-adic integers to the set of maximal ideals $\operatorname{Max}\left(C\left(\mathbb{Z}_{p}, k\right)\right)$ of $C\left(\mathbb{Z}_{p}, k\right)$ given by

$$
\mathbb{Z}_{p} \stackrel{\epsilon}{\rightarrow} \operatorname{Max}\left(C\left(\mathbb{Z}_{p}, k\right)\right), z \mapsto \operatorname{ker}\left(e v_{z}\right)
$$

is a bijection.

Proof (1) The projection to the $e$-th coefficient in the $p$-adic expansion is a continuous map from $\mathbb{Z}_{p}$ to $\mathbb{F}_{p}$. It is thus continuous as a function from $\mathbb{Z}_{p}$ to $k$. Moreover it coincides with $\left(\begin{array}{c}s \\ p^{e}\end{array}\right)$ on $\mathbb{N}$ by Lucas' Theorem (Theorem 1.1.4). This provides the morphism $i$ of the statement. It is injective since $\mathbb{N}$ is dense in $\mathbb{Z}_{p}$. As the $\left(\begin{array}{c}s \\ p^{e}\end{array}\right)$ generate the algebra of binomial coefficients by Corollary 1.1.5, it is a direct consequence of Mahler's Theorem, see e.g. [16, III 1.2.4], that $i$ is surjective. 
(2) Since $\left(\begin{array}{c}s \\ p^{e}\end{array}\right)$ is the projection to the $e$-th coordinate in the $p$-adic expansion, it is clear that the map $\mathbb{Z}_{p} \stackrel{\epsilon}{\rightarrow} \operatorname{Max}\left(C\left(\mathbb{Z}_{p}, k\right)\right)$ is injective. Indeed if $z=\Sigma_{e \geq 0} z_{e} p^{e}$ is different from $z^{\prime}=\Sigma_{e \geq 0} z_{e}^{\prime} p^{e}$, then there is a number $l \geq 0$ such that $z_{l}$ is different from $z_{l}^{\prime}$. Thus $\left(\begin{array}{c}s \\ p^{l}\end{array}\right)-z_{l}$ belongs to $\operatorname{ker}\left(e v_{z}\right)$ but not to $\operatorname{ker}\left(e v_{z^{\prime}}\right)$.

Let us show that $\epsilon$ is surjective. Let $z=\Sigma_{e \geq 0} z_{e} p^{e}$, the maximal ideal $\operatorname{ker}\left(e v_{z}\right)$ is generated by $\left\{\left(\begin{array}{c}s \\ p^{e}\end{array}\right)-z_{e} ; e \geq 0\right\}$. Indeed the set $\left\{\left(\begin{array}{c}s \\ p^{e}\end{array}\right)-z_{e} ; e \geq 0\right\}$ is contained in $\operatorname{ker}\left(e v_{z}\right)$, and since the ideal generated by $\left\{\left(\begin{array}{c}s \\ p^{e}\end{array}\right)-z_{e} ; e \geq 0\right\}$ is obviously maximal, it has to be equal to $\operatorname{ker}\left(e v_{z}\right)$. Let $\mathfrak{m}$ be a maximal ideal of $C\left(\mathbb{Z}_{p}, k\right)$ and let

$$
C\left(\mathbb{Z}_{p}, k\right) \stackrel{m}{\rightarrow} K:=C\left(\mathbb{Z}_{p}, k\right) / \mathfrak{m}
$$

be the quotient morphism. Since $\left(\begin{array}{c}s \\ p^{e}\end{array}\right)^{p}=\left(\begin{array}{c}s \\ p^{e}\end{array}\right), m\left(\left(\begin{array}{c}s \\ p^{e}\end{array}\right)\right)$ is in the prime field $\mathbb{F}_{p}$, for all $e$. Let $a_{e}$ be the lift of $m\left(\left(\begin{array}{c}s \\ p^{e}\end{array}\right)\right)$ to $\{0, \ldots, p-1\}$ and let $a=\Sigma_{e \geq 0} a_{e} p^{e}$. The maximal ideal $\mathfrak{m}$ contains $k e r\left(e v_{a}\right)$. They are thus equal. This shows that $\epsilon$ is surjective and concludes the proof of the theorem.

Let us single out a special word for the vanishing locus of an ideal.

Definition 1.1.9 Let $I$ be an ideal of the $k$-algebra of binomial coefficients. The roots of $I$ are the $p$-adic integers corresponding by Theorem 1.1.8 to the maximal ideals containing $I$.

The following proposition shows that the naive notion of multiplicity is not so interesting for the algebra of binomial coefficients.

Proposition 1.1.10 (1) The k-algebra of binomial coefficients is a Jacobson ring.

(2) The ideals of $\mathbb{F} p\left[\left(\begin{array}{c}s \\ p^{0}\end{array}\right),\left(\begin{array}{c}s \\ p^{1}\end{array}\right),\left(\begin{array}{c}s \\ p^{2}\end{array}\right), \ldots\right]$ are radical. They are thus characterised by their sets of roots.

Proof (1) Let $A$ denote the $k$-algebra of binomial coefficients. Let us show that every finitely generated $A$-algebra that is a field is a finitely generated $A$-module. This is one of the characterisations of Jacobson rings, see e.g. [1, Ch. 5 Exercise 25]. Let $K$ be such a field and let $v$ be the homomorphism of $k$-algebras

$$
A \stackrel{v}{\rightarrow} K, a \mapsto a .1 .
$$

Since $\left(\begin{array}{c}s \\ p^{e}\end{array}\right)^{p}=\left(\begin{array}{c}s \\ p^{e}\end{array}\right)$ for all $e \geq 0$, its image by $v$ is in the prime field and accordingly $v(A)=k$. It is well-known that fields are Jacobson rings. Therefore $K$ is a finitely generated $k$-module. Hence it is a finitely generated $A$-module. We have thus proved that $A$ is a Jacobson ring.

(2) Let $f$ be an element of $\mathbb{F}_{p}\left[\left(\begin{array}{c}s \\ p^{0}\end{array}\right),\left(\begin{array}{c}s \\ p^{1}\end{array}\right),\left(\begin{array}{c}s \\ p^{2}\end{array}\right), \ldots\right] \cong C\left(\mathbb{Z}_{p}, \mathbb{F}_{p}\right)$. Clearly $f^{p^{n}}=f$, for all natural numbers $n$. Suppose that $f$ is in the radical of an ideal $I$, i.e. $f^{N}$ is in $I$ for some $N \geq 0$. Let $n \geq 0$ be such that $p^{n} \geq N$. We have that $f=f^{p^{n}}$ is in $I$. Thus the ideal $I$ is radical. Since by (1), the algebra of binomial coefficients is radical, we have that every radical ideal is the intersection of maximal ideals. Hence every ideal of $\mathbb{F}_{p}\left[\left(\begin{array}{c}s \\ p^{0}\end{array}\right),\left(\begin{array}{c}s \\ p^{1}\end{array}\right),\left(\begin{array}{c}s \\ p^{2}\end{array}\right), \ldots\right]$ is the intersection of the maximal ideals containing it. It is thus characterised by its set of roots. 


\subsection{Definition of the $b$-function}

Let $k$ be a field of characteristic $p>0$ and let $R$ be a commutative $k$-algebra. Recall that we denote by $D_{R}$ the ring of Grothendieck differential operators of $R$ over $k$.

Definition 1.2.1 Let $R$ be a smooth commutative $k$-algebra and let $f$ be an element of $R$. Denote by $R[t]$ the ring of polynomials in one variable $t$ over $R$.

(1) There is an inclusion of left $D_{R[t]}$-modules $R[t] \subset R[t]\left[\frac{1}{f-t}\right]$. Let $B_{f}$ be the quotient left $D_{R}[t]$-module $R[t]\left[\frac{1}{f-t}\right] / R[t]$.

(2) Let $D_{R}\left[v_{e} ; e \in \mathbb{N}\right]$ be the subring of $D_{R[t]}$ generated by $D_{R}$ and the higher Euler operators $v_{e}:=\frac{d}{d t}^{\left[p^{e}\right]} t^{p^{e}}$, for all $e \geq 0$. The delta function of $f$ is the class $\delta_{f}$ of $\frac{1}{f-t}$ in $B_{f}$. Let $M_{f}$ be the left $D_{R}\left[v_{e} ; e \in \mathbb{N}\right]$-submodule of $B_{f}$ generated by $\delta_{f}$.

Lemma 1.2.2 For all natural numbers $e$, we have the following identity in $D_{\mathbb{F}_{p}[t]}$ :

$$
\left[v_{e}, t\right]=t \Pi_{j=0}^{j=e-1}\left(1-v_{j}^{p-1}\right),
$$

where the product over the empty set, i.e. for $e=0$, is 1 . In particular, the $k$-algebra of higher Euler operators $\Gamma_{k}$ satisfies $\Gamma_{k} t=t \Gamma_{k}$ in $D_{k[t]}$.

Proof For the identity to hold in $D_{\mathbb{F}_{p}[t]}$, it is enough that both sides agree when evaluated at monomials $t^{N}$, for all natural numbers $N \geq 0$.

One easily sees that, for the left-hand side:

$$
\left[v_{e}, t\right]\left(t^{N}\right):=\left(v_{e} t-t v_{e}\right)\left(t^{N}\right)=\left(\begin{array}{c}
N \\
p^{e}-1
\end{array}\right) t^{N+1}
$$

and for the right-hand side:

$$
t \prod_{j=0}^{j=e-1}\left(1-v_{j}^{p-1}\right)\left(t^{N}\right)=\prod_{j=0}^{j=e-1}\left(1-\left(\left(\begin{array}{c}
N \\
p^{j}
\end{array}\right)+1\right)^{p-1}\right) t^{N+1}
$$

Since $n^{p-1}=1 \bmod p$ if and only if $n \neq 0 \bmod p$, one has that

$$
\Pi_{j=0}^{j=e-1}\left(1-\left(\left(\begin{array}{l}
N \\
p^{j}
\end{array}\right)+1\right)^{p-1}\right)=0 \bmod p
$$

if and only if there is a number $j$ in $\{0, \ldots, e-1\}$ such that $\left(\begin{array}{l}N \\ p^{j}\end{array}\right) \neq p-1 \bmod p$. By Lucas' Theorem, this is the case if and only if

$$
N \neq \Sigma_{j=0}^{j=e-1}(p-1) p^{j} \bmod p^{e} .
$$


Since $\Sigma_{j=0}^{j=e-1}(p-1) p^{j}=p^{e}-1$, it is thus clear using Lucas' Theorem again that both sides of the identity evaluated at $t^{N}$ vanish if $N \neq p^{e}-1 \bmod p^{e}$ and that they are equal to $t^{N+1}$, otherwise. This concludes the proof of the identity.

The inclusion $\Gamma_{k} t \subset t \Gamma_{k}$ follows immediately from the identity. The reverse inclusion $t \Gamma_{k} \subset \Gamma_{k} t$ follows from the identity and an easy induction. Namely, it suffices to show that for all natural numbers $m, n_{0}, \ldots, n_{m}$, the monomial $t v_{m}^{n_{m}} \ldots v_{0}^{n_{0}}$ is in $\Gamma_{k} t$. Let us well-order the finite sequences $\left(m, n_{m}, \ldots, n_{0}\right)$ by the lexicographic order and induct on it. It is clear that for the smallest element $(0,0), t v_{0}^{0}=t$ is in $\Gamma_{k} t$. Consider a sequence $\left(m, n_{m}, \ldots, n_{0}\right)$. If $n_{m}=0$, then $t v_{m}^{n_{m}} \ldots v_{0}^{n_{0}}=$ $t v_{m-1}^{n_{m-1}} \ldots v_{0}^{n_{0}}$, corresponding to the smaller $\left(m-1, n_{m-1}, \ldots, n_{0}\right)$. It is thus in $\Gamma_{k} t$ by the induction hypothesis. Suppose that $n_{m} \geq 1$, then by the identity, $\begin{aligned} t v_{m}^{n_{m}} \ldots v_{0}^{n_{0}} & =t v_{m} v_{m}^{n_{m}-1} \ldots v_{0}^{n_{0}} \\ & =v_{m} t v_{m}^{n_{m}-1} \ldots v_{0}^{n_{0}}-t \Pi_{j=0}^{j=m-1}\left(1-v_{j}^{p-1}\right) v_{m}^{n_{m}-1} \ldots v_{0}^{n_{0}},\end{aligned}$

which is in $\Gamma_{k} t$ by the induction hypothesis. This concludes the proof of the lemma.

Corollary 1.2.3 The left $D_{R}$-submodule $t M_{f}$ of $M_{f}$ is stable under the action of the higher Euler operators. Hence the quotient $N_{f}:=M_{f} / t M_{f}$ is a left $D_{R}\left[v_{e} ; e \in \mathbb{N}\right]$ module.

Proof By Lemma 1.2.2, $\Gamma_{k} t M_{f}=t \Gamma_{k} M_{f}=t M_{f}$.

We can now give the definition of the $b$-function.

Definition 1.2.4 Let $k$ be a field of characteristic $p>0, R$ a smooth $k$-algebra and let $f$ be an element of $R$. The left $D_{R}\left[v_{e} ; e \in \mathbb{N}\right]$-module $N_{f}$ is in particular a $\Gamma_{k}$-module. Recall that we fixed in Lemma 1.1.6 an isomorphism

$$
k\left[\left(\begin{array}{c}
s \\
p^{0}
\end{array}\right),\left(\begin{array}{c}
s \\
p^{1}
\end{array}\right),\left(\begin{array}{c}
s \\
p^{2}
\end{array}\right), \ldots\right] \stackrel{\gamma}{\rightarrow} \Gamma_{k} .
$$

We denote by $N_{f}^{\gamma}$ the $k\left[\left(\begin{array}{c}s \\ p^{0}\end{array}\right),\left(\begin{array}{c}s \\ p^{1}\end{array}\right),\left(\begin{array}{c}s \\ p^{2}\end{array}\right), \ldots\right]$-module deduced from $N_{f}$ by the isomorphism $\gamma$. The $b$-function $b_{f}$ of $f$ is the annihilator of $N_{f}^{\gamma}$ in $k\left[\left(\begin{array}{c}s \\ p^{0}\end{array}\right),\left(\begin{array}{c}s \\ p^{1}\end{array}\right),\left(\begin{array}{c}s \\ p^{2}\end{array}\right), \ldots\right]$.

Remark 1.2.5 We note that the definitions of $B_{f}, M_{f}$, and hence of $N_{f}$, are local on $\operatorname{Spec}(R)$. This allows us to globalise the definition of the $b$-function to $f$ a function on a smooth $k$-variety, for example. We leave the details to the reader.

\subsection{Bounded level versions of $N_{f}$}

Here we start analysing $N_{f}$ by considering differential operators of bounded level. Let us recall some definitions. From here on, let us suppose that the base field $k$ is perfect.

Definition 1.3.1 Let $p$ be a prime number. Let $R$ be a commutative algebra of characteristic $p$. The Frobenius endomorphism of $R$ is the ring endomorphism $F$ of $R$ raising elements to their $p$-th power,

$$
\begin{aligned}
& R \stackrel{F}{\rightarrow} R, \\
& r \mapsto r^{p} .
\end{aligned}
$$


Definition 1.3.2 Let $k$ be a perfect field of characteristic $p>0, R$ a smooth $k$-algebra and $n$ a natural number $\geq 0$. Let $R^{(n+1)}$ be the $R$-module $R \stackrel{F^{n+1}}{\rightarrow} R$. The ring $D_{R}^{(n)}$ of differential operators of level $n$ on $\operatorname{Spec}(R)$ is the ring of $R$-linear endomorphisms of $R^{(n+1)}$.

Proposition 1.3.3 Let $l$ be a natural number. The differential operators of level l are differential operators. Namely the canonical inclusion of $D_{R}^{(l)}$ in $\operatorname{End}_{k}(R)$ factors through $D_{R}$. Moreover, $D_{R}=\bigcup_{n \geq 0} D_{R}^{(n)}$.

Proof This is well-known. For example, it follows from [5, Proposition 2.2.7] and [5, (2.2.1.7)]

We clearly have that the differential operators of level $n$ in $D_{R}\left[v_{e} ; e \in \mathbb{N}\right]$ are $D_{R[t]}^{(n)} \cap D_{R}\left[v_{e} ; e \in \mathbb{N}\right]=D_{R}^{(n)}\left[v_{e} ; 0 \leq e \leq n\right]$. It is thus natural to consider the subsequent bounded level versions of $M_{f}$ and $N_{f}$.

Definition 1.3.4 Let $l$ be a natural number. The left $D_{R}^{(l)}\left[v_{e} ; 0 \leq e \leq l\right]$-module $M_{f}^{(l)}$ is

$$
M_{f}^{(l)}:=D_{R}^{(l)}\left[v_{e} ; 0 \leq e \leq l\right] \delta_{f} \subset M_{f} .
$$

By Lemma 1.2.2, we have that the left $D_{R}^{(l)}$-submodule $t M_{f}^{(l)}$ of $M_{f}^{(l)}$ is a left $D_{R}^{(l)}\left[v_{e} ; 0 \leq e \leq l\right]$-submodule. The left $D_{R}^{(l)}\left[v_{e} ; 0 \leq e \leq l\right]$-module $N_{f}^{(l)}$ is set to be the quotient,

$$
N_{f}^{(l)}:=M_{f}^{(l)} / t M_{f}^{(l)}
$$

Let $l$ be a natural number and let $M$ be a $R$-module. By functoriality of the tensor product $-\otimes_{R} M$, the Frobenius pull-back $R$-module $\left(F^{l+1}\right)^{*} M:=R^{(l+1)} \otimes_{R} M$ is acted upon on the left by the ring of $R$-linear endomorphisms of $R^{(l+1)}$, i.e. by $D_{R}^{(l)}$. The Frobenius pull-back is actually an equivalence of categories.

Proposition 1.3.5 Let $k$ be a perfect field of characteristic $p>0$ and $R$ a smooth $k$-algebra. Let $l$ be a natural number. The Frobenius pull-back $\left(F^{l+1}\right)^{*}$ induces an equivalence from the category of finitely generated $R$-modules to the category of finitely generated left $D_{R}^{(l)}$-modules.

Proof This is a well-known instance of a Morita equivalence. For example, see [23, Proposition 3.2.(1)].

Section 1.5 is devoted to describing a preimage of $N_{f}^{(l)}$ under this equivalence.

\subsection{Test ideals}

In this subsection and the next, unless otherwise mentioned, we use the following notations: $k$ is a perfect field of characteristic $p>0, R$ is a smooth $k$-algebra and $f$ is an element of $R$. 
Let us recall the definition of the test ideals of $f$ and sum up the properties we will use.

Definition 1.4.1 Let $e$ be a natural number and $J$ an ideal of $R$. The $p^{e}$-th root ideal $J^{\left[\frac{1}{p^{e}}\right]}$ of $J$ is the intersection of all the ideals $I$ of $R$ such that $I^{\left[p^{e}\right]} \supset J$, where $I^{\left[p^{e}\right]}$ is the ideal of $R$ generated by the $p^{e}$-th powers of the elements of $I$.

Lemma 1.4.2 Let $\lambda$ be a non-negative real number and e a natural number. There is an inclusion of ideals of $R$,

$$
\left(f^{\left\lceil\lambda p^{e}\right\rceil} R\right)^{\left[\frac{1}{p^{e}}\right]} \subset\left(f^{\left\lceil\lambda p^{e+1}\right\rceil} R\right)^{\left[\frac{1}{p^{e+1}}\right]},
$$

where $\rceil$ is the ceiling function which maps a real number to the smallest greater integer.

Proof The lemma is a special case of [6, Lemma 2.8].

Definition 1.4.3 Let $\lambda$ be a non-negative real number. The test ideal of exponent $\lambda$ of $f$ is the following ideal of $R$,

$$
\tau\left(f^{\lambda}\right):=\bigcup_{e \geq 0}\left(f^{\left\lceil\lambda p^{e}\right\rceil} R\right)^{\left[\frac{1}{p^{e}}\right]} .
$$

The test ideal of exponent $\lambda$ of $f$ clearly decreases as $\lambda$ increases.

Lemma 1.4.4 Let $\lambda$ be a non-negative real number. There is a real number $\epsilon>0$ such that the test ideal of exponent $\lambda^{\prime}$ of $f$ is $\tau\left(f^{\lambda}\right)$, for every non-negative real number $\lambda^{\prime}$ in the interval $[\lambda, \lambda+\epsilon)$.

Proof This is a special case of [6, Corollary 2.16].

Definition 1.4.5 A $F$-jumping exponent of $f$ is a positive real number $\lambda$ such that for all real numbers $\epsilon>0$ such that the difference $\lambda-\epsilon$ is positive, $\tau\left(f^{\lambda-\epsilon}\right) \neq \tau\left(f^{\lambda}\right)$.

The following finiteness result is crucial to us.

Theorem 1.4.6 The set of $F$-jumping exponents of $f$ is

(1) a discrete subset of $\mathbb{R}$

(2) a subset of $\mathbb{Q}$.

Proof This is [6, Theorem 3.1], for a principal ideal.

We will also use the following definition.

Definition 1.4.7 Let $\lambda$ be a positive real number. The test ideal immediately preceding $\tau\left(f^{\lambda}\right)$ is $\tau\left(f^{\lambda^{-}}\right):=\cap_{\mu<\lambda} \tau\left(f^{\mu}\right)$. The associated graded to the test ideal filtration of $R$ with respect to $f$ is

$$
\mathfrak{g r}_{\lambda \in(0,1]}\left(\tau\left(f^{\lambda}\right)\right):=\bigoplus_{\lambda \in(0,1]} \tau\left(f^{\lambda^{-}}\right) / \tau\left(f^{\lambda}\right) .
$$


Lemma 1.4.8 Let $\lambda$ be a positive real number. Denote by $\lambda^{\prime}$ the supremum of the subset $S_{\lambda}$ of the real numbers containing 0 and the $F$-jumping exponents of $f$ strictly smaller than $\lambda$. Since $S_{\lambda}$ is discrete by (1) of Theorem 1.4.6, $\lambda^{\prime}$ is in $S_{\lambda}$.

The test ideal immediately preceding $\tau\left(f^{\lambda}\right)$ is $\tau\left(f^{\lambda}\right)$ if and only if $\lambda$ is not a $F$-jumping exponent of $f$. If $\lambda$ is a F-jumping exponent of $f$, then the test ideal immediately preceding $\tau\left(f^{\lambda}\right)$ is $\tau\left(f^{\lambda^{\prime}}\right)$.

Proof If $\lambda$ is not a $F$-jumping exponent, then there exists a real number $\epsilon>0$ such that $\tau\left(f^{\lambda-\epsilon}\right)=\tau\left(f^{\lambda}\right)$. Since the filtration by test ideals is decreasing, $\tau\left(f^{\lambda^{-}}\right):=$ $\cap_{\mu<\lambda} \tau\left(f^{\mu}\right)=\tau\left(f^{\lambda}\right)$.

If $\lambda$ is a $F$-jumping exponent, then for all real numbers $r$ in $\left[\lambda^{\prime}, \lambda\right), \tau\left(f^{r}\right)=\tau\left(f^{\lambda^{\prime}}\right)$. Thus $\tau\left(f^{\lambda^{-}}\right)=\tau\left(f^{\lambda^{\prime}}\right)$.

Corollary 1.4.9 Let $\left\{\lambda_{1}<\cdots<\lambda_{n}\right\}$ be the $F$-jumping exponents of $f$ in the interval $(0,1]$. For all integers $i$ in $\{1, \ldots, n\}$, the test ideal immediately preceding $\tau\left(f^{\lambda_{i}}\right)$ is $\tau\left(f^{\lambda_{i-1}}\right)$, where we have set $\lambda_{0}=0$. Thus the non-zero summands of the associated graded to the test ideal filtration of $R$ with respect to $f$ are indexed by the $F$-jumping exponents of $f$ in the interval $(0,1]$ and

$$
\mathfrak{g r}_{\lambda \in(0,1]}\left(\tau\left(f^{\lambda}\right)\right)=\bigoplus_{i=1}^{i=n} \tau\left(f^{\lambda_{i-1}}\right) / \tau\left(f^{\lambda_{i}}\right)
$$

Proof It follows directly from Lemma 1.4.8

\section{$1.5 N_{f}^{(l)}$ and test ideals}

By explicit computations, Mustaţă observed the following.

Proposition 1.5.1 Let $k$ be a perfect field of characteristic $p>0, R$ a smooth $k$ algebra and let $f$ be an element of $R$. Let $l$ be a natural number.

(1) There is a natural isomorphism of left $D_{R}^{(l)}$-modules,

$$
M_{f}^{(l)} \cong \bigoplus_{0 \leq n<p^{l+1}} D_{R}^{(l)} f^{n}
$$

where for all elements $r$ of $R, D_{R}^{(l)} r$ is the left $D_{R}^{(l)}$-submodule of $R$ generated by $r$. It induces an isomorphism $N_{f}^{(l)} \cong \bigoplus_{0 \leq n<p^{l+1}} D_{R}^{(l)} f^{n} / D_{R}^{(l)} f^{n+1}$.

(2) Let $n^{\prime}$ be a natural number $<p^{l+1}$ and let $n^{\prime}=\sum_{0 \leq e \leq l} a_{e} p^{e}$ be its base $p$ expansion. The action of the higher Euler operators on $N_{f}^{(l)}$ transported by the isomorphism of (1) to an action on $\bigoplus_{0 \leq n<p^{l+1}} D_{R}^{(l)} f^{n} / D_{R}^{(l)} f^{n+1}$ stabilises $D_{R}^{(l)} f^{n^{\prime}} / D_{R}^{(l)} f^{n^{\prime}+1}$ and for all $e$ in $\{0, \ldots, l\}, v_{e}$ acts on $D_{R}^{(l)} f^{n^{\prime}} / D_{R}^{(l)} f^{n^{\prime}+1}$ by $-a_{e}$. 
(3) The natural inclusion $M_{f}^{(l)} \subset M_{f}^{(l+1)}$ is transported by the isomorphisms of level $l$ and $l+1$ of (1) to

$$
\begin{aligned}
& \bigoplus_{0 \leq n<p^{l+1}} D_{R}^{(l)} f^{n} \rightarrow \bigoplus_{0 \leq m<p^{l+2}} D_{R}^{(l+1)} f^{m} \\
& \sum_{0 \leq n<p^{l+1}} g_{n} \mapsto \sum_{0 \leq n<p^{l+1}} \sum_{0 \leq j<p}(-1)^{j}\left(\begin{array}{c}
p-1 \\
j
\end{array}\right)\left(g_{n} f^{j p^{l+1}}\right)_{n+j p^{l+1}},
\end{aligned}
$$

where the subscript of an element indicates the direct summand to which it belongs.

Proof (1) The first isomorphism is shown in [20, Proposition 6.1] and the second in [20, Corollary 6.5].

(2) See [20, Corollary 6.5].

(3) It is [20, Remark 5.7].

Recall that we want to express a preimage of $N_{f}^{(l)}$ under the Frobenius pull-back $\left(F^{l+1}\right)^{*}$. We start with $D_{R}^{(l)} f^{n}$.

Lemma 1.5.2 There is a canonical isomorphism of left $D_{R}^{(l)}$-modules coming from an elementary equality of ideals,

$$
D_{R}^{(l)} f^{n} \cong\left(F^{l+1}\right)^{*} \tau\left(f^{\frac{n}{p^{l+1}}}\right) .
$$

Proof By [7, Lemma 2.2], the left $D_{R}^{(l)}$-submodule of $R$ generated by $f^{n}$ is

$$
\left(\left(f^{n}\right)^{\left[\frac{1}{p^{l+1}}\right]}\right)^{\left[p^{l+1}\right]}
$$

The latter is none other than $\tau\left(f^{\frac{n}{p^{l+1}}}\right)^{\left[p^{l+1}\right]} \cong\left(F^{l+1}\right)^{*} \tau\left(f^{\frac{n}{p^{l+1}}}\right)$, by [7, Lemma 2.1].

Definition 1.5.3 Let $l$ be a natural number. We say that $l$ separates the $F$-jumping exponents of $f$ if the partition of $(0,1]$ in intervals of length $\frac{1}{p^{l+1}}$ separates the $F$-jumping exponents of $f$, i.e. for each natural number $n<p^{l+1}$, the interval $\left(\frac{n}{p^{l+1}}, \frac{n+1}{p^{l+1}}\right]$ contains at most one $F$-jumping exponent of $f$.

Proposition 1.5.4 Let $l$ be a natural number. Recall that by (1) of Theorem 1.4.6, there are only finitely many $F$-jumping exponents of $f$ in the unit interval $[0,1]$ and suppose that $l$ is large enough to separate the $F$-jumping exponents of $f$. Then the canonical isomorphism of Lemma 1.5.2 induces an isomorphism of left $D_{X}^{(l)}$-modules,

$$
N_{f}^{(l)} \cong\left(F^{l+1}\right)^{*}\left(\mathfrak{g r}_{\lambda \in(0,1]}\left(\tau\left(f^{\lambda}\right)\right)\right)
$$


Hence for every natural number $l^{\prime}$ greater than $l$, the map induced from that in (3) of Proposition 1.5.1 transports to a morphism

$$
\left(F^{l^{\prime}+1}\right)^{*}\left(\mathfrak{g r}_{\lambda \in(0,1]}\left(\tau\left(f^{\lambda}\right)\right)\right) \stackrel{a_{l^{\prime}+1}}{\rightarrow}\left(F^{l^{\prime}+2}\right)^{*}\left(\mathfrak{g r}_{\lambda \in(0,1]}\left(\tau\left(f^{\lambda}\right)\right)\right)
$$

and there is an isomorphism of left $D_{R}$-modules

$$
N_{f}=\underset{l^{\prime} \geq l}{\lim } N_{f}^{\left(l^{\prime}\right)} \cong \underset{l^{\prime} \geq l}{\lim }\left(F^{l^{\prime}+1}\right)^{*}\left(\mathfrak{g r}_{\lambda \in(0,1]}\left(\tau\left(f^{\lambda}\right)\right)\right),
$$

where $\lim _{l^{\prime} \geq l}\left(F^{l^{\prime}+1}\right)^{*}\left(\mathfrak{g r}_{\lambda \in(0,1]}\left(\tau\left(f^{\lambda}\right)\right)\right)$ denotes the direct limit with respect to these morphisms.

Proof By (1) of Proposition 1.5.1 and Lemma 1.5.2, for all natural numbers $l$, we have that

$$
N_{f}^{(l)} \cong \bigoplus_{0 \leq n<p^{l+1}}\left(F^{l+1}\right)^{*} \tau\left(f^{\frac{n}{p^{l+1}}}\right) /\left(F^{l+1}\right)^{*} \tau\left(f^{\frac{n+1}{p^{l+1}}}\right)
$$

and thus that

$$
N_{f}^{(l)} \cong\left(F^{l+1}\right)^{*}\left(\bigoplus_{0 \leq n<p^{l+1}} \tau\left(f^{\frac{n}{p^{l+1}}}\right) / \tau\left(f^{\frac{n+1}{p^{l+1}}}\right)\right)
$$

by flatness of the Frobenius pull-back.

Let $n$ be a natural number $<p^{l+1}$. If there is no $F$-jumping exponent of $f$ in the interval $\left(\frac{n}{p^{l+1}}, \frac{n+1}{p^{l+1}}\right]$, then $\tau\left(f^{\frac{n}{p^{l+1}}}\right)=\tau\left(f^{\frac{n+1}{p^{l+1}}}\right)$. Thus the quotient $\tau\left(f^{\frac{n}{p^{l+1}}}\right) / \tau\left(f^{\frac{n+1}{p^{l+1}}}\right)$ vanishes. On the other hand, if there is an $F$-jumping exponent of $f$ in the interval $\left(\frac{n}{p^{l+1}}, \frac{n+1}{p^{l+1}}\right]$, then the quotient $\tau\left(f^{\frac{n}{p^{l+1}}}\right) / \tau\left(f^{\frac{n+1}{p^{l+1}}}\right)$ is equal to $\tau\left(f^{\lambda^{-}}\right) / \tau\left(f^{\lambda}\right)$, where $\lambda$ is the $F$-jumping coefficient in $\left(\frac{n}{p^{l+1}}, \frac{n+1}{p^{l+1}}\right]$, unique by the hypothesis on $l$.

Finally, since, for varying $n$, the intervals $\left(\frac{n}{p^{l+1}}, \frac{n+1}{p^{l+1}}\right]$ form a partition of $(0,1]$, all $F$-jumping coefficients of $f$ in $(0,1]$ appear in this way. Thus one has that

$$
\left.\bigoplus_{0 \leq n<p^{l+1}} \tau\left(f^{\frac{n}{p^{l+1}}}\right) / \tau\left(f^{\frac{n+1}{p^{l+1}}}\right)\right)=\bigoplus_{\lambda} \tau\left(f^{\lambda^{-}}\right) / \tau\left(f^{\lambda}\right),
$$

where the direct sum on the right-hand side is over the $F$-jumping exponents of $f$ in the interval $(0,1]$. One applies Corollary 1.4 .9 to conclude the proof of the proposition.

In the next section, we use the relation between $N_{f}$ and the $F$-jumping exponents of $f$ to get information about the roots of its $b$-function. 


\section{Relation to test ideals and rationality of the roots}

\subsection{Preliminaries on $p$-adic and $\frac{1}{p}$-adic expansions}

We will express the $p$-adic expansions of the roots of the $b$-function of $f$ in terms of the " $\frac{1}{p}$-adic expansions" of its $F$-jumping exponents. Let us precise what we mean.

Definition 2.1.1 Let $p$ be a prime number and let $r$ be a positive real number. The $\frac{1}{p}$-adic expansion of the number $r$ is its unique base $p$ expansion which does not have infinitely many consecutive zero coefficients. It is written $r=\sum_{-b \leq n} r_{n}\left(\frac{1}{p}\right)^{n}$, where the coefficients $r_{n}$ belong to the set $\{0, \ldots, p-1\}$ and $b$ is a natural number. The $\frac{1}{p}$-adic fractional part of $r$ is $\operatorname{frac}_{\frac{1}{p}}(r):=\sum_{n \geq 1} r_{n}\left(\frac{1}{p}\right)^{n}$ and its $\frac{1}{p}$-adic integer part is $[r]_{\frac{1}{p}}:=\sum_{n=-b}^{n=0} r_{n}\left(\frac{1}{p}\right)^{n}$.

Definition 2.1.2 A positive real number $r$ of $\frac{1}{p}$-adic expansion $r=\sum_{-b \leq n} r_{n}\left(\frac{1}{p}\right)^{n}$ is $\frac{1}{p}$-adically periodic if there is a natural number $n^{\prime} \geq 1$ such that the sequence $\left(r_{n}\right)_{n \geq n^{\prime}}$ is repeating. Let $n^{\prime \prime}$ be the smallest such natural number. The $\frac{1}{p}$-adic anteperiod of $r$ is $n^{\prime \prime}-1$. The $\frac{1}{p}$-adic period of $r$ is the period $\rho_{r}$ of the sequence $\left(r_{n}\right)_{n \geq n^{\prime \prime}}$ and the word $r_{n^{\prime \prime}} \ldots r_{n^{\prime \prime}+\rho_{r}-1}$ is its $\frac{1}{p}$-adic repetend. A $\frac{1}{p}$-adically periodic number is said to be purely $\frac{1}{p}$-adically periodic if its $\frac{1}{p}$-adic anteperiod is 0 .

The following lemma characterises the periodicity properties of $\frac{1}{p}$-adic expansions.

\section{Lemma 2.1.3 Let $p$ be a prime number.}

(1) A positive real number is $\frac{1}{p}$-adically periodic if and only if it is rational.

(2) A positive real number is purely $\frac{1}{p}$-adically periodic if and only if it is in $\mathbb{Z}_{(p)}$.

Proof (1) To prove that $\frac{1}{p}$-adic periodicity implies rationality, one notices that for all $e \geq 1, \sum_{n>0} \frac{1}{p^{e n}}=\frac{1}{p^{e}-1}$. The rest of the proof is very standard and left to the reader.

(2) The assertion is invariant under shifts by natural numbers. Hence it suffices to prove it for positive real numbers in the interval $(0,1]$.

If a number $r \in(0,1]$ is purely $\frac{1}{p}$-adically periodic, then its $\frac{1}{p}$-adic expansion is of the form $r_{0} \sum_{n>0} \frac{1}{p^{e n}}=\frac{r_{0}}{p^{e}-1}$, where $e$ is a natural number $>1$ and $r_{0}$ is smaller than $p^{e}$. Thus $r$ is in $\mathbb{Z}_{(p)}$.

Suppose that $r$ is in $\mathbb{Z}_{(p)} \cap(0,1]$ and let $r=\frac{a}{b}$ be its reduced rational expression. Then it is well-known that, since $b$ is prime to $p$, there is a positive natural number $e$ such that $b$ divides $p^{e}-1$. Thus $r=\frac{a^{\prime}}{p^{e}-1}=a^{\prime} \sum_{n>0} \frac{1}{p^{e n}}$, with $0<a^{\prime}<p^{e}$. Hence $r$ is purely $\frac{1}{p}$-adically periodic. bers.

To each $\frac{1}{p}$-adically periodic number correspond finitely many "conjugated" num- 
Definition 2.1.4 The $\frac{1}{p}$-adic conjugates of a $\frac{1}{p}$-adically periodic number $r$ are the numbers whose $\frac{1}{p}$-adic expansion is obtained from that of $r$ by a cyclic permutation of the letters of its $\frac{1}{p}$-adic repetend.

Thus for $l$ the anteperiod of $r$ and $d$ its period, the $\frac{1}{p}$-adic conjugates of $r=$ $\frac{b_{1}}{p}+\cdots+\frac{b_{l}}{p^{l}}+\frac{a_{1}}{p^{l+1}}+\frac{a_{2}}{p^{l+2}}+\cdots+\frac{a_{d-1}}{p^{l+d-1}}+\frac{a_{d}}{p^{l+d}}+\frac{a_{1}}{p^{l+d+1}}+\cdots$ are

$$
\begin{aligned}
& \left\{\frac{b_{1}}{p}+\cdots+\frac{b_{l}}{p^{l}}+\frac{a_{1}}{p^{l+1}}+\frac{a_{2}}{p^{l+2}}+\cdots+\frac{a_{d-1}}{p^{l+d-1}}+\frac{a_{d}}{p^{l+d}}+\frac{a_{1}}{p^{l+d+1}}+\ldots,\right. \\
& \frac{b_{1}}{p}+\cdots+\frac{b_{l}}{p^{l}}+\frac{a_{d}}{p^{l+1}}+\frac{a_{1}}{p^{l+2}}+\cdots+\frac{a_{d-2}}{p^{l+d-2}}+\frac{a_{d-1}}{p^{l+d}}+\frac{a_{d}}{p^{l+d+1}}+\ldots, \ldots, \\
& \left.\frac{b_{1}}{p}+\cdots+\frac{b_{l}}{p^{l}}+\frac{a_{2}}{p^{l+1}}+\frac{a_{3}}{p^{l+2}}+\cdots+\frac{a_{d}}{p^{l+d-1}}+\frac{a_{1}}{p^{l+d}}+\frac{a_{2}}{p^{l+d+1}}+\cdots\right\} \text {. }
\end{aligned}
$$

We now present a pure periodicity lemma which will be useful in our description of the roots of the $b$-function. We start with a definition.

Definition 2.1.5 Let $n$ be a positive natural number and let $a_{1}, \ldots, a_{n}$ be elements of $\{0, \ldots, p-1\}$. Set $a$ to be the rational number $a:=\sum_{i=1}^{i=n} \frac{a_{i}}{p^{i}}$. A positive real number $\lambda$ begins with $a$ if its $\frac{1}{p}$-adic fractional part begins with $a$, i.e. if there are coefficients $\lambda_{j}$ for all $\mathrm{j}$ greater than $\mathrm{n}$ such that

$$
\operatorname{frac}_{\frac{1}{p}}(\lambda)=\sum_{i=1}^{i=n} \frac{a_{i}}{p^{i}}+\sum_{j>n} \frac{\lambda_{j}}{p^{j}} .
$$

Lemma 2.1.6 Let $s$ be a sequence $s: \mathbb{N}_{0} \rightarrow\{0, \ldots, p-1\}, n \mapsto s(n)$ and let $\Lambda$ be a finite set of rational numbers. For all positive natural numbers $l$, set $s_{l}:=$ $\sum_{i=1}^{i=l} \frac{s(l-i+1)}{p^{i}}$.

Suppose that for all natural numbers $N$ large enough, there is an element $\lambda$ of $\Lambda$ beginning with $s_{N}$. Then there is a number $L$ such that for all $n$ greater than $L$, there is a purely $\frac{1}{p}$-adically periodic element of $\Lambda$ beginning with $s_{n}$.

Proof Since $\Lambda$ is a finite set, there is a number $L$ greater than $N$ such that for each $l$ greater than $L$, there is an element $\lambda$ in $\Lambda$ beginning with $s_{l}$ and beginning with $s_{l^{\prime}}$ for infinitely many $l^{\prime}$ greater than $l$. Let us prove that such a rational number $\lambda$ has to be purely $\frac{1}{p}$-adically periodic.

Clearly, one may suppose that $\lambda$ coincides with its $\frac{1}{p}$-adic fractional part. Let $\lambda=$ $\sum_{j \geq 1} \frac{\lambda_{j}}{p^{j}}$ be its $\frac{1}{p}$-adic expansion. By (1) of Lemma $2.1 .3, \lambda$ is $\frac{1}{p}$-adically periodic. Let $n$ be its anteperiod and $\rho>0$ be its period. We claim that for all numbers $j \geq 1, \lambda_{j+\rho}=\lambda_{j}$. This indeed implies that $\lambda$ is purely $\frac{1}{p}$-adically periodic. We will show that the hypothesis on the beginning of $\lambda$ allows one to embed the beginning of its $\frac{1}{p}$-adic expansion into its repeating part, thus forcing pure periodicity. 
More precisely, since $\lambda_{j+\rho}=\lambda_{j}$ for all $j>n$ by definition of the anteperiod $n$, it is enough to show that $\lambda_{j+\rho}=\lambda_{j}$ for all $1 \leq j \leq n$. Consider $l$ greater than $\max \{n+\rho, L\}$ such that $\lambda$ begins by $s_{l}$. Thus for all $1 \leq i \leq n+\rho, \lambda_{i}$ is equal to $s(l-i+1)$. Since there is also $l^{\prime}$ greater than $l+n$ such that $\lambda$ begins with $s_{l^{\prime}}$, we have that for all $1 \leq i \leq n$,

$$
\lambda_{i}=s(l-i+1)=s\left(l^{\prime}-\left(l^{\prime}-l+i\right)+1\right)=\lambda_{l^{\prime}-l+i} .
$$

Moreover $l^{\prime}-l$ is not smaller than the anteperiod $n$, thus $l^{\prime}-l+i>n$. Hence $\lambda_{l^{\prime}-l+i}$ is equal to $\lambda_{l^{\prime}-l+i+\rho}$. Using again that $\lambda$ begins with $s_{l^{\prime}}$, we get that the latter $\lambda_{l^{\prime}-l+i+\rho}=s\left(l^{\prime}-\left(l^{\prime}-l+i+\rho\right)+1\right)=s(l-(i+\rho)+1)$. Recall that $\lambda$ begins also with $s_{l}$ and thus that $s(l-(i+\rho)+1)=\lambda_{i+\rho}$. In conclusion, we have proved that for each $i$ between 1 and $n$,

$$
\lambda_{i}=\lambda_{l^{\prime}-l+i}=\lambda_{l^{\prime}-l+i+\rho}=\lambda_{i+\rho} .
$$

This completes the proof of the lemma.

Finally, let us express the $p$-adic expansion of a number in $\mathbb{Z}_{(p)}$ in terms of the $\frac{1}{p}$-adic expansion of its opposite. Note that a positive real number is in the interval $(0,1]$ if and only if it equals its $\frac{1}{p}$-adic fractional part. We have the following.

Lemma 2.1.7 Let $r$ be a positive real number in the interval $(0,1]$. Suppose that $r$ is purely $\frac{1}{p}$-adically periodic and let $r=\sum_{n \geq 1} \frac{r_{n}}{p^{n}}$ be its $\frac{1}{p}$-adic expansion. Let $d$ be its $\frac{1}{p}$-adic period. Then the $p$-adic expansion of $-r$ is

$$
-r=\sum_{n \geq 0} r_{d-n_{d}} p^{n}
$$

where $n_{d}$ is the representative of $n \bmod d$ in $\{0, \ldots, d-1\}$.

Proof Let $d$ be the $\frac{1}{p}$-adic period of $r$ and let $r^{\prime}:=\sum_{i=1}^{i=d} r_{i} p^{d-i}$. By pure periodicity, we have that $r=r^{\prime} \sum_{e \geq 1} \frac{1}{p^{d e}}$. Moreover $\sum_{e \geq 1} \frac{1}{p^{d e}}=\frac{1}{p^{d}-1}$ and the $p$-adic expansion of $-\frac{1}{p^{d}-1}$ is $\sum_{j \geq 0} p^{d j}$. Thus the $p$-adic expansion of $-r$ is equal to

$$
-r=r^{\prime} \sum_{j \geq 0} p^{d j}=\sum_{1 \leq i \leq d ; j \geq 0} r_{i} p^{d j+d-i}=\sum_{n \geq 0} r_{d-n_{d}} p^{n}
$$

\subsection{Unit $F$-modules}

In the next subsection, we will show that $N_{f}$ seen as a left $D_{R}$-module is of a very particular type, namely it is a unit $F$-module. Let us first recall their definition and basic properties. 
From now on, unless otherwise mentioned, $k$ is a perfect field of characteristic $p>$ $0, R$ is a smooth $k$-algebra, $f$ is an element of $R$ and $F$ is the Frobenius endomorphism of $R$.

Definition 2.2.1 Let $M$ be an $R$-module and $e$ a natural number. Let $R \stackrel{\mu_{M}}{\rightarrow} \operatorname{End}_{k}(M)$ be the structure map. The $e$-th Frobenius direct image of $M$ is the $R$-module $F_{*}^{e} M$ whose underlying $k$-vector space is $M$, with structure map $\mu_{M} \circ F^{e}: R \stackrel{F^{e}}{\rightarrow} R \stackrel{\mu_{M}}{\rightarrow}$ $\operatorname{End}_{k}(M)$.

Definition 2.2.2 Let $M$ be an $R$-module and $e$ be a positive integer. An $F^{e}$-module structure on $M$ is an $R$-linear morphism $F_{M}^{e}: M \rightarrow F_{*}^{e} M$. If we do not want to specify $e$, we call an $F^{e}$-module a Frobenius module or $F$-module.

Definition 2.2.3 Let $e$ be a positive integer. The ring $R\left[F^{e}\right]$ is the ring generated by $R$ and an element $F^{e}$, with the relations $F^{e} r=r^{p^{e}} F^{e}$, for all elements $r$ of $R$. The data of an $F^{e}$-module structure on an $R$-module is clearly equivalent to that of a left $R\left[F^{e}\right]$-module structure, compatible with the action of $R$. We say that an $F^{e}$-module is finitely generated if it is so as a left $R\left[F^{e}\right]$-module.

Definition 2.2.4 Let $e$ be a positive integer. An $F^{e}$-module $M$ is unit if the adjoint map to the structure map $F_{M}^{e}$,

$$
\left(F^{e}\right)^{*} M \stackrel{F_{M}^{e}{ }^{\prime}}{\rightarrow} M
$$

is an isomorphism.

The following is well-known.

Proposition 2.2.5 Let $e$ be a positive integer. A unit $F^{e}$-module is canonically endowed with a structure of left $D_{R}$-module.

Proof Denote the unit $F^{e}$-module by $M$. For all natural numbers $n$, we have that $\gamma_{n}:=\left(F^{n e}\right)^{*}\left(F_{M}^{e}\right) \circ \cdots \circ\left(F^{e}\right)^{*}\left(F_{M}^{e^{\prime}}\right) \circ F_{M}^{e^{\prime}}$ is an $R$-linear isomorphism $\left(F^{(n+1) e}\right)^{*} M \stackrel{\gamma_{n}}{\rightarrow} M$. As noted in Sect. $1.3,\left(F^{(n+1) e}\right)^{*} M$ is endowed with a canonical left $D_{R}^{((n+1) e-1)}$-module structure. Hence so is $M$, via the isomorphism $\gamma_{n}$. Moreover, it is straightforward to check that those actions are compatible with the inclusions $D_{R}^{((n+1) e-1)} \subset D_{R}^{((n+2) e-1)}$. Since $D_{R}=\bigcup_{n \geq 0} D_{R}^{((n+1) e-1)}, M$ is thus canonically a left $D_{R}$-module.

There is a convenient way to generate unit $F$-modules, due to Lyubeznik [18].

Proposition 2.2.6 Let $e$ be a positive integer. Let $G$ be an $R$-module and $\beta$ be an $R$-linear morphism $G \stackrel{\beta}{\rightarrow}\left(F^{e}\right)^{*} G$. Consider the direct limit of the direct system

$$
G \stackrel{\beta}{\rightarrow}\left(F^{e}\right)^{*} G \stackrel{\left(F^{e}\right)^{*} \beta}{\rightarrow}\left(F^{2 e}\right)^{*} G{\stackrel{\left(F^{2 e}\right.}{\rightarrow}}^{*} \beta
$$

and denote it $\lim _{\longrightarrow} \beta$. Then the $R$-module $\lim _{\longrightarrow} \beta$ is naturally isomorphic to its pull-back

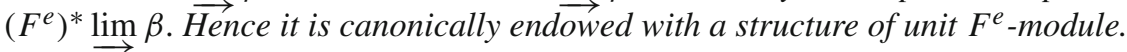


Proof This is a direct consequence of the commutation of pull-back with direct limits.

Definition 2.2.7 Let $e$ be a positive integer and $M$ a unit $F^{e}$-module. The data of a finitely generated $R$-module $G$, an $R$-linear morphism $G \stackrel{\beta}{\rightarrow}\left(F^{e}\right)^{*} G$ and an isomorphism $\iota$ of Frobenius modules $\lim \beta \rightarrow M$ is called a generator of $M$. We will often omit to mention the isomorphism $\iota$.

In fact, all finitely generated unit $F$-modules appear this way, as noted by EmertonKisin.

Theorem 2.2.8 Every finitely generated unit $F^{e}$-module has a generator.

Proof This is a special case of [9, Theorem 6.1.3].

Here is a fundamental finiteness property of unit $F$-modules by which they stand out among $D_{R}$-modules. It is due to Lyubeznik.

Theorem 2.2.9 A finitely generated unit $F$-module is of finite length as a left $D_{R^{-}}$ module.

Proof It follows immediately from [18, Theorem 3.2] and [18, Theorem 5.6].

\subsection{A unit $F$-structure on $N_{f}$}

Here we continue the study of $N_{f}$ via its bounded level versions started in Sect. 1.5. In particular, we show that the left $D_{R}$-module $N_{f}$ is a unit $F$-module and the higher Euler operators are compatible with its Frobenius endomorphism.

Proposition 2.3.1 Let $l$ be a natural number and $\lambda$ a $F$-jumping exponent of $f$ in $(0,1]$. Suppose that $l$ is large enough to separate the $F$-jumping exponents of $f$. Then the composition of the isomorphisms of Propositions 1.5.1(1) and 1.5.4,

$$
\bigoplus_{0 \leq n<p^{l+1}} D_{R}^{(l)} f^{n} / D_{R}^{(l)} f^{n+1} \cong N_{f}^{(l)} \cong\left(F^{l+1}\right)^{*}\left(\mathfrak{g r}_{\lambda \in(0,1]}\left(\tau\left(f^{\lambda}\right)\right)\right)
$$

induces an isomorphism

$$
D_{R}^{(l)} f^{m} / D_{R}^{(l)} f^{m+1} \cong\left(F^{l+1}\right)^{*}\left(\tau\left(f^{\lambda^{-}}\right) / \tau\left(f^{\lambda}\right)\right)
$$

for the unique $m$ such that $\frac{m}{p^{l+1}}$ is the truncated $\frac{1}{p}$-adic expansion of $\lambda$.

Proof It is clear that $\lambda \in\left(\frac{n}{p^{l+1}}, \frac{n+1}{p^{l+1}}\right]$ if and only if the $\frac{1}{p}$-adic expansion of $\lambda$ begins with $\frac{n}{p^{l+1}}$. The proposition follows then easily from the definition of the isomorphism of Proposition 1.5.4. 
Let us now study the structure maps of the inductive system for $N_{f}$ from Proposition 1.5.4 in more details.

Proposition 2.3.2 There are natural numbers $L$ and $N$ such that for all $F$-jumping exponents $\lambda$ of $f$ in $(0,1]$ which are not in $\mathbb{Z}_{(p)}$, and for all $l>L$ and $e>N$, the restriction to $\left(F^{l+1}\right)^{*}\left(\tau\left(f^{\lambda^{-}}\right) / \tau\left(f^{\lambda}\right)\right)$ of the e-th iterate of the structure maps

$$
\left(F^{l+1}\right)^{*}\left(\mathfrak{g r}_{\mu \in(0,1]}\left(\tau\left(f^{\mu}\right)\right)\right) \rightarrow\left(F^{l+e+1}\right)^{*}\left(\mathfrak{g r}_{\mu \in(0,1]}\left(\tau\left(f^{\mu}\right)\right)\right)
$$

vanishes.

Proof We first take $L$ large enough so that $l$ separates the $F$-jumping exponents of $f$. By Proposition 2.3.1 and the description of the structure maps in (3) of Proposition 1.5.1, for all positive $i$, the $i$-th iterate of the structure maps restricts to

$$
\left(F^{l+1}\right)^{*}\left(\tau\left(f^{\lambda^{-}}\right) / \tau\left(f^{\lambda}\right)\right) \rightarrow \bigoplus_{0 \leq n<p^{l+i+1} \text { and } n=m \bmod p^{l+1}} D_{R}^{(l+i)} f^{n} / D_{R}^{(l+i)} f^{n+1},
$$

for the unique $m$ such that $\frac{m}{p^{l+1}}$ is the truncated $\frac{1}{p}$-adic expansion of $\lambda$.

We claim that there are numbers $N$ and $L$ such that if $\lambda \notin \mathbb{Z}_{(p)}$, then for all $i>N$, and $l>L$, the above map vanishes.

Let us argue by contradiction and suppose that there are $l$ and $i$, large at will, such that the above map does not vanish. In particular the target of the map is not trivial. Hence there is a number $n_{i}$, congruent to $m$ modulo $p^{l+1}$, such that $\frac{n_{i}}{p^{l+i+1}}$ is the truncated $\frac{1}{p}$-adic expansion of an $F$-jumping exponent of $f$. Thus $n_{i}=m+b_{i} p^{l+1}$, for a certain natural number $b_{i}=\Sigma_{j=0}^{j=i-1}\left(b_{i}\right)_{j+1} p^{j}$ and

$$
\frac{n_{i}}{p^{l+i+1}}=\frac{\left(b_{i}\right)_{i}}{p}+\frac{\left(b_{i}\right)_{i-1}}{p^{2}}+\cdots+\frac{\left(b_{i}\right)_{1}}{p^{i}}+\frac{m_{1}}{p^{i+1}}+\frac{m_{2}}{p^{i+2}}+\cdots+\frac{m_{l+1}}{p^{i+l+1}},
$$

where $\frac{m_{1}}{p}+\frac{m_{2}}{p^{2}}+\cdots+\frac{m_{l+1}}{p^{l+1}}$ is the truncated $\frac{1}{p}$-adic expansion of $\lambda$. Since the map is an $i$-th iterate, for it not to vanish it is necessary that the corresponding $j$-th iterate does not vanish either, for all $j \leq i$. Hence one may assume that as $i$ varies, the numbers $b_{i}$ are compatible with each others. Namely for all $i \leq i^{\prime}$ such that $b_{i}$ and $b_{i^{\prime}}$ are defined, one has that $b_{i^{\prime}}$ is congruent to $b_{i}$ modulo $p^{i}$. For all $1 \leq j \leq i$, let us denote $(b)_{j}$ the coefficient $\left(b_{i}\right)_{j}=\left(b_{i^{\prime}}\right)_{j}$.

For every $l$ as above, we define the sequence $s:=\left(m_{l+1}, m_{l}, \ldots, m_{1},(b)_{1}\right.$, $\left.(b)_{2}, \ldots\right)$. Let $\Lambda$ be the set of $F$-jumping exponents of $f$ in $(0,1]$. It is clear that for all $n$ large enough, there is an element of $\Lambda$ beginning with $s_{n}$, using the notation of Lemma 2.1.6. Hence by Lemma 2.1.6, for $n$ large enough, there is a purely $\frac{1}{p}$-adically periodic element of $\Lambda$ beginning with $s_{n}$. As $l$ can be taken as large as one wishes, this implies that the sequence $\left(m_{1}, m_{2}, \ldots\right)$ is purely periodic, that is that $\lambda$ is purely $\frac{1}{p}$-adically periodic. This is absurd since $\lambda$ is in the complement of $\mathbb{Z}_{(p)}$, by hypothesis. We have thus shown the required vanishing. 
This allows us to give an inductive system for the $D_{R}$-module $N_{f}$ expressed only in terms of the $F$-jumping exponents of $f$ in $(0,1] \cap \mathbb{Z}_{(p)}$. Recall from Proposition 1.5.4 that for all $l$ large enough to separate the $F$-jumping exponents of $f$, we have maps:

$$
\left(F^{l+1}\right)^{*}\left(\mathfrak{g r}_{\lambda \in(0,1]}\left(\tau\left(f^{\lambda}\right)\right)\right) \stackrel{a_{l+1}}{\rightarrow}\left(F^{l+2}\right)^{*}\left(\mathfrak{g r}_{\lambda \in(0,1]}\left(\tau\left(f^{\lambda}\right)\right)\right)
$$

We set

$$
\left(F^{l+1}\right)^{*}\left(\mathfrak{g r}_{\lambda \in(0,1] \cap \mathbb{Z}_{(p)}}\left(\tau\left(f^{\lambda}\right)\right)\right) \stackrel{b_{l+1}}{\rightarrow}\left(F^{l+2}\right)^{*}\left(\mathfrak{g r}_{\lambda \in(0,1] \cap \mathbb{Z}_{(p)}}\left(\tau\left(f^{\lambda}\right)\right)\right)
$$

to be $b_{l+1}:=\left(F^{l+2}\right)^{*}(\pi) \circ a_{l+1} \circ\left(F^{l+1}\right)^{*}(i)$, where $i$ is the natural injection

$$
\mathfrak{g r}_{\lambda \in(0,1] \cap \mathbb{Z}_{(p)}}\left(\tau\left(f^{\lambda}\right)\right) \stackrel{i}{\rightarrow} \mathfrak{g r}_{\lambda \in(0,1]}\left(\tau\left(f^{\lambda}\right)\right)
$$

and $\pi$ is the natural projection $\mathfrak{g r}_{\lambda \in(0,1]}\left(\tau\left(f^{\lambda}\right)\right) \stackrel{\pi}{\rightarrow} \mathfrak{g r}_{\lambda \in(0,1] \cap \mathbb{Z}_{(p)}}\left(\tau\left(f^{\lambda}\right)\right)$. We also have to consider the following intermediate system:

$$
\left(F^{l+1}\right)^{*}\left(\mathfrak{g r}_{\lambda \in(0,1]}\left(\tau\left(f^{\lambda}\right)\right)\right) \stackrel{c_{l+1}}{\rightarrow}\left(F^{l+2}\right)^{*}\left(\mathfrak{g r}_{\lambda \in(0,1]}\left(\tau\left(f^{\lambda}\right)\right)\right)
$$

with $c_{l+1}:=\left(F^{l+2}\right)^{*}(i) \circ\left(F^{l+2}\right)^{*}(\pi) \circ a_{l+1}$. We will denote the corresponding inductive systems by $\left(a_{j}\right),\left(b_{j}\right)$ and $\left(c_{j}\right)$, respectively.

Corollary 2.3.3 On one hand, the injections $\left(F^{j}\right)^{*}(i)$ give rise to a morphism of inductive systems $\left(b_{j}\right) \rightarrow\left(c_{j}\right)$. Denote it $\bar{i}$. On the other, the maps

$$
\left(F^{j}\right)^{*}\left(\mathfrak{g r}_{\lambda \in(0,1]}\left(\tau\left(f^{\lambda}\right)\right)\right) \stackrel{I d}{\rightarrow}\left(F^{j}\right)^{*}\left(\mathfrak{g r}_{\lambda \in(0,1]}\left(\tau\left(f^{\lambda}\right)\right)\right)
$$

and

$$
\left(F^{j+1}\right)^{*}\left(\mathfrak{g r}_{\lambda \in(0,1]}\left(\tau\left(f^{\lambda}\right)\right)\right) \stackrel{\left(F^{j+1}\right)^{*}(i) \circ\left(F^{j+1}\right)^{*}(\pi)}{\rightarrow}\left(F^{j+1}\right)^{*}\left(\mathfrak{g r}_{\lambda \in(0,1]}\left(\tau\left(f^{\lambda}\right)\right)\right)
$$

induce a morphism $\left(a_{j}\right) \rightarrow\left(c_{j}\right)$. Let us denote it $\bar{\pi}$.

The morphisms $\bar{i}$ and $\bar{\pi}$ induce isomorphisms of direct limits. In particular $N_{f}$ is isomorphic to the direct limit of $\left(b_{j}\right), \lim _{\longrightarrow}\left(F^{j}\right)^{*}\left(\mathfrak{g r}_{\lambda \in(0,1] \cap \mathbb{Z}_{(p)}}\left(\tau\left(f^{\lambda}\right)\right)\right)$.

Proof It is a straightforward consequence of Proposition 2.3.2.

We can now prove the

Theorem 2.3.4 Let $k$ be a perfect field of characteristic $p>0, R$ a smooth $k$-algebra and let $f$ be an element of $R$. 
(1) Let $e$ be the lcm of the lengths of the periods of the $\frac{1}{p}$-adic expansions of the $F$-jumping exponents of $f$ in $(0,1] \cap \mathbb{Z}_{(p)}$. The left $D_{R}$-module $N_{f}$ is a finitely generated unit $F^{e}$-module.

(2) For eachl separating the $F$-jumping exponents of $f$, the unit $F^{e}$-module $N_{f}$ splits as a direct sum

$$
N_{f}=\bigoplus_{\lambda \in(0,1] \cap \mathbb{Z}_{(p)}}\left(N_{f}\right)_{\lambda}
$$

where the $\lambda$ are the $F$-jumping exponents of $f$ in $(0,1] \cap \mathbb{Z}_{(p)}$. Each summand is a direct limit

$$
\left(N_{f}\right)_{\lambda}:=\lim _{j \geq 0}\left(F^{l+1+e j}\right)^{*}\left(\tau\left(f^{\lambda^{-}}\right) / \tau\left(f^{\lambda}\right)\right)
$$

where the limit is for the maps induced by the system $\left(b_{j}\right)$ and it is non-trivial,

$$
\left(N_{f}\right)_{\lambda} \neq 0
$$

(3) The higher Euler operators act as endomorphisms of the unit $F^{e}$-module $N_{f}$.

Proof (1) We use the inductive system $\left(b_{j}\right)$ of Corollary 2.3.3, which by that Corollary has $N_{f}$ as direct limit. Pick an $l$ that separates the $F$-jumping exponents of $f$ and denote $\beta_{e}$ the morphism $b_{l+e} \circ \cdots \circ b_{l+2} \circ b_{l+1}$ :

$$
\left(F^{l+1}\right)^{*}\left(\mathfrak{g r}_{\mu \in(0,1] \cap \mathbb{Z}_{(p)}}\left(\tau\left(f^{\mu}\right)\right)\right) \stackrel{\beta_{e}}{\rightarrow}\left(F^{e}\right)^{*}\left(F^{l+1}\right)^{*}\left(\mathfrak{g r}_{\mu \in(0,1] \cap \mathbb{Z}_{(p)}}\left(\tau\left(f^{\mu}\right)\right)\right) .
$$

We claim that $\beta_{e}$ is a generator. It is enough to check that for all $r \geq 0$, the pull-back $\left(F^{r e}\right)^{*}\left(\beta_{e}\right)$ coincides with the composition of the structure maps from $\left(b_{j}\right)$, that is $\left(F^{r e}\right)^{*}\left(\beta_{e}\right)=b_{(r+1) e+l} \circ \cdots \circ b_{r e+l+1}$. We see by the description of the structure maps from Proposition 1.5.1.3 and Proposition 2.3.1 that for all purely $\frac{1}{p}$-adically periodic $F$-jumping exponent $\lambda$, both $\beta_{e, r}:=b_{(r+1) e+l} \circ \cdots \circ b_{r e+l+1}$ and $\left(F^{r e}\right)^{*}\left(\beta_{e}\right)$ send

$$
\left(F^{r e+l+1}\right)^{*}\left(\tau\left(f^{\lambda^{-}}\right) / \tau\left(f^{\lambda}\right)\right) \text { to }\left(F^{(r+1) e+l+1}\right)^{*}\left(\tau\left(f^{\lambda^{-}}\right) / \tau\left(f^{\lambda}\right)\right) \text {. }
$$

Indeed let $\frac{m}{p^{l+1}}$ be the truncated $\frac{1}{p}$-adic expansion of $\lambda$. Since $e$ is a multiple of the periods, they both have to factor through the sum of the factors indexed by $F$-jumping exponents $\mu$ with the same truncated $\frac{1}{p}$-adic expansion $\frac{m}{p^{l+1}}$. But $l$ separates the $F$ jumping exponents hence $\mu$ has to be equal to $\lambda$.

Moreover if $\frac{m}{p^{l+1}}+\frac{p^{l+1} n}{p^{e+l+1}}$ is the truncated $\frac{1}{p}$-adic expansion of $\lambda$, we have by the description of the structure maps that $\beta_{e, r}$ is the multiplication by $c f^{p^{r e+l+1} n}$, for a non-zero element $c$ of $\mathbb{F}_{p}$. We thus have that $\beta_{e, r}=\left(F^{r e}\right)^{*}\left(\beta_{e, 0}\right)$. Since $\beta_{e, 0}$ is equal to $\beta_{e}$, this concludes the proof of the point. 
(2) The existence of the decomposition and the description of each summand as a direct limit follow directly from the description of $\left(b_{j}\right)$ in the proof of the point (1) above.

Let us prove the non-triviality of the summands, $\left(N_{f}\right)_{\lambda} \neq 0$. Fix an $F$-jumping exponent $\lambda$ of $f$ in $(0,1] \cap \mathbb{Z}_{(p)}$. By Proposition 2.3.1 and the direct limit description of the summands, the vanishing $\left(N_{f}\right)_{\lambda}=0$ implies that the image under the structure map of $f^{m} \in D_{R}^{(l)} f^{m}$ in $D_{R}^{(l+j e)} f^{m^{\prime}} / D_{R}^{(l+j e)} f^{m^{\prime}+1}$ is zero, for some positive $j$, and $m$ and $m^{\prime}$ such that $\frac{m}{p^{l+1}}$ and $\frac{m^{\prime}}{p^{l+j e+1}}$ are the truncated $\frac{1}{p}$-adic expansions of $\lambda$. But by Proposition 1.5.1(3), the image of $f^{m}$ is a non-zero scalar times $f^{m^{\prime}}$. Thus its vanishing implies that of the quotient:

$$
0=D_{R}^{(l+j e)} f^{m^{\prime}} / D_{R}^{(l+j e)} f^{m^{\prime}+1} \cong\left(F^{l+j e+1}\right)^{*}\left(\tau\left(f^{\frac{m^{\prime}}{p^{l+j e+1}}}\right) / \tau\left(f^{\frac{m^{\prime}+1}{p^{l+j e+1}}}\right)\right)
$$

and hence that of

$$
\tau\left(f^{\frac{m^{\prime}}{p^{l+j e+1}}}\right) / \tau\left(f^{\frac{m^{\prime}+1}{p^{l+j e+1}}}\right)=0 .
$$

Which by the definition of $m^{\prime}$ is absurd since $\lambda$ is a $F$-jumping exponent of $f$.

(3) By (2) it is enough to show the result for each of the unit $F^{e}$-modules $\left(N_{f}\right)_{\lambda}$. Moreover by definition of the isomorphisms in Corollary 2.3.3, the action of the higher Euler operators induced on the image of $\left(F^{l+1+e j}\right)^{*}\left(\tau\left(f^{\lambda^{-}}\right) / \tau\left(f^{\lambda}\right)\right)$ in the direct limit of $\left(b_{j}\right)$ coincides with that induced by its image in the direct limit of $\left(a_{j}\right)$. It is thus given, via Proposition 2.3.1, by Proposition 1.5.1.2. That is the image of $\left(F^{l+1+e j}\right)^{*}\left(\tau\left(f^{\lambda^{-}}\right) / \tau\left(f^{\lambda}\right)\right)$ is a common eigenspace of $\left\{v_{0}, v_{1}, \ldots, v_{l+e j}\right\}$ of eigenvalue $-m_{i}$ for $\nu_{i}$, where $\frac{\Sigma_{i=0}^{i=l+e j} m_{i} p^{i}}{p^{l+1+e j}}$ is the truncated $\frac{1}{p}$-adic expansion of $\lambda$. Since $e$ is a multiple of the $\frac{1}{p}$-adic period of $\lambda$, we have that if $m_{i+e}$ is defined, then $m_{i+e}=m_{i}$. In particular for all numbers $i$ and $j^{\prime}$ such that $0 \leq i \leq l+j^{\prime} e$, the action of $v_{i}$ on the image of $\left(F^{l+1+e j^{\prime}}\right)^{*}\left(\tau\left(f^{\lambda^{-}}\right) / \tau\left(f^{\lambda}\right)\right)$ in $N_{f}$ is given by the same number $-m_{i}$. The action of the higher Euler operators is thus compatible with the $F^{e}$-module structure.

Remark 2.3.5 The decomposition of $N_{f}$ as a direct $\operatorname{sum} N_{f}=\bigoplus_{\lambda \in(0,1] \cap \mathbb{Z}_{(p)}}\left(N_{f}\right)_{\lambda}$ is canonical. However, the assignment of a $F$-jumping exponent to each of the summands is not. Indeed, different choices of $l$ in (2) of Theorem 2.3.4 exchange the $\left(N_{f}\right)_{\lambda}$ 's, for $\frac{1}{p}$-conjugated $\lambda$ 's. This only depends on $l \bmod e$.

We now briefly prove that the $b$-function has finitely many roots using the RiemannHilbert correspondence for unit $F$-modules [9]. This will be reproved in Theorem 2.4.1 which will provide more precise information about the roots.

Corollary 2.3.6 Let $k$ be a perfect field of characteristic $p>0, R$ a smooth $k$-algebra and let $f$ be an element of $R$. The $b$-function of $f$ has finitely many roots. 
Proof We show that there are only finitely many maximal ideals of

$$
k\left[\left(\begin{array}{c}
s \\
p^{0}
\end{array}\right),\left(\begin{array}{c}
s \\
p^{1}
\end{array}\right),\left(\begin{array}{c}
s \\
p^{2}
\end{array}\right), \ldots\right]
$$

containing the ideal $b_{f} \subset k\left[\left(\begin{array}{c}s \\ p^{0}\end{array}\right),\left(\begin{array}{c}s \\ p^{1}\end{array}\right),\left(\begin{array}{c}s \\ p^{2}\end{array}\right), \ldots\right]$. It is an immediate consequence of Theorem 1.1.8 that the maximal ideals of $k\left[\left(\begin{array}{c}s \\ p^{0}\end{array}\right),\left(\begin{array}{c}s \\ p^{1}\end{array}\right),\left(\begin{array}{c}s \\ p^{2}\end{array}\right), \ldots\right]$ are defined over $\mathbb{F}_{p}$. Thus it is enough to show that there are only finitely many maximal ideals of $\mathbb{F}_{p}\left[\left(\begin{array}{c}s \\ p^{0}\end{array}\right),\left(\begin{array}{c}s \\ p^{1}\end{array}\right),\left(\begin{array}{c}s \\ p^{2}\end{array}\right), \ldots\right]$ containing $b_{f} \cap \mathbb{F}_{p}\left[\left(\begin{array}{c}s \\ p^{0}\end{array}\right),\left(\begin{array}{c}s \\ p^{1}\end{array}\right),\left(\begin{array}{c}s \\ p^{2}\end{array}\right), \ldots\right]$. The latter is the annihilator of $N_{f}^{\gamma}$ in $\mathbb{F}_{p}\left[\left(\begin{array}{c}s \\ p^{0}\end{array}\right),\left(\begin{array}{c}s \\ p^{1}\end{array}\right),\left(\begin{array}{c}s \\ p^{2}\end{array}\right), \ldots\right]$.

By the Riemann-Hilbert correspondence for unit $F$-modules [9, Theorem 11.4.2], the unit $F^{e}$-module $N_{f}$ corresponds to an object in the constructible derived category of étale $\mathbb{F}_{p}$-sheaves on $\operatorname{Spec}(R)$. Since by (3) of Theorem 2.3.4, the algebra

$$
\mathbb{F}_{p}\left[\left(\begin{array}{c}
s \\
p^{0}
\end{array}\right),\left(\begin{array}{c}
s \\
p^{1}
\end{array}\right),\left(\begin{array}{c}
s \\
p^{2}
\end{array}\right), \ldots\right]
$$

acts on $N_{f}$ by endomorphisms of unit $F^{e}$-module, it is transported by the RiemannHilbert correspondence to act on an object in the constructible derived category of étale $\mathbb{F}_{p}$-sheaves on $\operatorname{Spec}(R)$. The algebra of global endomorphims of those being finite dimensional over $\mathbb{F}_{p}$, the algebra $\mathbb{F}_{p}\left[\left(\begin{array}{c}s \\ p^{0}\end{array}\right),\left(\begin{array}{c}s \\ p^{1}\end{array}\right),\left(\begin{array}{c}s \\ p^{2}\end{array}\right), \ldots\right] / b_{f}$ is finite dimensional over $\mathbb{F}_{p}$. It has thus finitely many maximal ideals, which proves the corollary.

\subsection{The roots of the $b$-function and $F$-jumping exponents}

Let us now describe the roots of the $b$-function of $f$ in terms of its $F$-jumping exponents.

Theorem 2.4.1 Let $k$ be a perfect field of characteristic $p>0, R$ a smooth $k$-algebra and let $f$ be an element of $R$ not contained in $k .1$. The roots of the b-function of $f$ are the opposites of the $F$-jumping exponents of $f$ which are in $(0,1] \cap \mathbb{Z}_{(p)}$.

Proof By Theorem 2.3.4,

$$
b_{f}:=a_{k} n_{k\left[\left(\begin{array}{c}
s \\
p^{0}
\end{array}\right),\left(\begin{array}{c}
s \\
p^{1}
\end{array}\right),\left(\begin{array}{c}
s \\
p^{2}
\end{array}\right), \ldots\right]}\left(N_{f}^{\gamma}\right)=\cap_{\lambda \in(0,1] \cap \mathbb{Z}_{(p)}} a n n_{k\left[\left(\begin{array}{c}
s \\
p^{0}
\end{array}\right),\left(\begin{array}{c}
s \\
p^{1}
\end{array}\right),\left(\begin{array}{c}
s \\
p^{2}
\end{array}\right), \ldots\right]}\left(N_{f}\right)_{\lambda}^{\gamma},
$$

where the $\lambda$ are the $F$-jumping exponents of $f$ in $(0,1] \cap \mathbb{Z}_{(p)}$ and the exponent $\gamma$ is a reminder that $k\left[\left(\begin{array}{c}s \\ p^{0}\end{array}\right),\left(\begin{array}{c}s \\ p^{1}\end{array}\right),\left(\begin{array}{c}s \\ p^{2}\end{array}\right), \ldots\right]$ acts via the isomorphism $\gamma$ of Lemma 1.1.6.

Let $\lambda$ be an $F$-jumping exponent of $f$ in $(0,1] \cap \mathbb{Z}_{(p)}$. Recall that the relation between the summand $\left(N_{f}\right)_{\lambda}$ of $N_{f}$ and the $F$-jumping exponent $\lambda$ depends on the choice of an integer $l$ separating the $F$-jumping exponents of $f$. For all $l$, we described the action of the higher Euler operators on $\left(N_{f}\right)_{\lambda}$ in the proof of Theorem 2.3.4.3. Namely the action of $\left(\begin{array}{c}s \\ p^{i}\end{array}\right)$ on $\left(N_{f}\right)_{\lambda}$ is the multiplication by $m_{i}$, where $\frac{\Sigma_{r=0}^{r=l+e j} m_{r} p^{r}}{p^{l+1+e j}}$ is the truncated $\frac{1}{p}$-adic expansion of $\lambda$, independently of $e$. Thus if the $\frac{1}{p}$-adic expansion 
of $\lambda$ is $\lambda=\Sigma_{n \geq 1} \frac{\lambda_{n}}{p^{n}}$, the annihilator of $\left(N_{f}\right)_{\lambda}^{\gamma}$ is the maximal ideal corresponding by Theorem 1.1.8 to the $p$-adic integer $\Sigma_{i \geq 0} m_{i} p^{i}$, with $m_{i}=\lambda_{i^{\prime}}$ for $i^{\prime}=l+1-i \bmod e$. The latter is well-defined since $m_{i+e}=m_{i}$ and $\lambda_{j+e}=\lambda_{j}$, for all $i, j$.

Hence for a choice of $l$ such that $l+1$ is a multiple of $e$, say $l+1=r e$, we have that the annihilator of $\left(N_{f}\right)_{\lambda}^{\gamma}$ is the maximal ideal corresponding to the $p$-adic integer $\Sigma_{i \geq 0} \lambda_{i^{\prime}} p^{i}$, where $i^{\prime}=r e-i \bmod e$. Which by Lemma 2.1.7 is equal to $\Sigma_{i \geq 0} \lambda_{i^{\prime}} p^{i}=-\lambda$.

Since we have in particular shown that the set of roots of the $b$-function of $f$ is the union for all $F$-jumping exponents $\mu$ of $f$ in $(0,1] \cap \mathbb{Z}_{(p)}$ of the $p$-adic integers corresponding to the annihilator of $\left(N_{f}\right)_{\mu}^{\gamma}$, this concludes the proof of the theorem.

Remark 2.4.2 Since there are only finitely many $F$-jumping exponents of $f$ in $(0,1]$ by Theorem 1.4.6, Theorem 2.4.1 implies Corollary 2.3.6, as announced.

The following is a direct consequence of Theorem 2.4.1.

Corollary 2.4.3 The roots of the $b$-function are negative rational numbers, $\geq-1$.

\section{Examples}

In this section, we use Theorem 2.4.1 to compute the roots of the $b$-function in some examples.

Example 3.0.4 Let $f=x$ in $\mathbb{F}_{p}[x]$. The set of roots of the $b$-function of $f$ is $\{-1\}$. Note that the Bernstein-Sato polynomial of $x$ in $\mathbb{C}[x]$ is $(s+1)$.

Example 3.0.5 Let $f=x_{1}^{2}+\cdots+x_{n}^{2}$ in $\mathbb{F}_{p}\left[x_{1}, \ldots, x_{n}\right]$, where $n \geq 2$ and $p>$ 2. The only $F$-jumping exponent of $f$ in $(0,1]$ is 1 [20, Example 6.16]. Thus by Theorem 2.4.1, the set of roots of the $b$-function of $f$ is $\{-1\}$. Note that the BernsteinSato polynomial of $x_{1}^{2}+\cdots+x_{n}^{2}$ in $\mathbb{C}\left[x_{1}, \ldots, x_{n}\right]$ is $\left(s+\frac{n}{2}\right)(s+1)$ [14, Example 6.2].

Example 3.0.6 For all $n$ and $j, 1 \leq j \leq n$, let $\alpha_{j}$ be a positive integer. It is well-known [12, Theorem 6.10] that the $F$-jumping exponents of the element $f=x_{1}^{\alpha_{1}} \ldots x_{n}^{\alpha_{n}}$ of $\mathbb{F}_{p}\left[x_{1}, \ldots, x_{n}\right]$ in $(0,1]$ are $\bigcup_{1 \leq j \leq n}\left\{\frac{l}{\alpha_{j}} \mid 1 \leq l \leq \alpha_{j}\right\}$. Thus by Theorem 2.4.1, the set of roots of the b-function of $f$ is

$$
\bigcup_{1 \leq j \leq n}\left\{-\frac{l}{\alpha_{j}} \mid 1 \leq l \leq \alpha_{j}\right\} \cap \mathbb{Z}_{(p)} .
$$

Note that by [14, Lemma 6.10], the Bernstein-Sato polynomial of the complex polynomial $x_{1}^{\alpha_{1}} \ldots x_{n}^{\alpha_{n}}$ is $\Pi_{1 \leq j \leq n} \Pi_{1 \leq l \leq \alpha_{j}}\left(s+\frac{l}{\alpha_{j}}\right)$.

Example 3.0.7 Suppose that $p$ is at least 5 and let $f_{p}=x^{2}+y^{3}$ in $\mathbb{F}_{p}[x, y]$. By [20, Example 6.14], the $F$-jumping exponents of $f_{p}$ in $(0,1]$ are: $\left\{\frac{5}{6}, 1\right\}$ if $p=1 \bmod 3$ and $\left\{\frac{5}{6}-\frac{1}{6 p}, 1\right\}$ if $p=2 \bmod 3$. Hence by Theorem 2.4.1, the roots of $b_{f_{p}}$ are:

$$
\left\{-1,-\frac{5}{6}\right\} \quad \text { if } \quad p=1 \bmod 3
$$


and

$$
\{-1\} \quad \text { if } \quad p=2 \bmod 3
$$

Note that by [14, Example 6.19], the Bernstein-Sato polynomial of $f=x^{2}+y^{3}$ is

$$
b_{f}=\left(s+\frac{7}{6}\right)(s+1)\left(s+\frac{5}{6}\right) \text {. }
$$

Example 3.0.8 Let $f_{p}=x^{5}+y^{4}+x^{3} y^{2}$ in $\mathbb{F}_{p}[x, y]$. By [21, Example 4.5], if $p=19 \bmod 20$, then $\frac{9 p-11}{20(p-1)}$ is a $F$-jumping exponent of $f_{p}$. Thus Theorem 2.4.1 implies that if $p=19 \bmod 20$, then

$$
-\frac{9 p-11}{20(p-1)}
$$

is a root of the $b$-function of $f_{p}$.

Acknowledgements This theory of the $b$-function in characteristic $p$ sprang from my attempt to understand [20]. I am indebted to Mircea Mustaţă for the work done there. I would also like to thank him for initially mentioning the problem and answering many questions about test ideals. I thank Konstantin Ardakov and Francesco Baldassarri for introducing me to Mahler's Theorem, thus clarifying the appearance of $\mathbb{Z}_{p}$ in the theory. Shunsuke Takagi kindly pointed me towards Example 3.0.8. Finally I would like to thank Roman Bezrukavnikov and Pavel Etingof for interesting discussions at the very beginning of this project. The author was partially supported by EPSRC Grant EP/L005190/1.

Open Access This article is distributed under the terms of the Creative Commons Attribution 4.0 International License (http://creativecommons.org/licenses/by/4.0/), which permits unrestricted use, distribution, and reproduction in any medium, provided you give appropriate credit to the original author(s) and the source, provide a link to the Creative Commons license, and indicate if changes were made.

\section{References}

1. Atiyah, M.F., Macdonald, I.G.: Introduction to Commutative Algebra. Addison-Wesley Publishing Co., Reading (1969)

2. Beřlinson, A.A.: How to glue perverse sheaves. In: $K$-Theory, Arithmetic and Geometry (Moscow, 1984-1986), vol. 1289 of Lecture Notes in Math., pp. 42-51. Springer, Berlin (1987)

3. Bernšteřn, I.N.: Analytic continuation of generalized functions with respect to a parameter. Funkcional. Anal. i Priložen. 6(4), 26-40 (1972)

4. Bernstein, J.: Algebraic theory of D-modules. Available at https://ncatlab.org/nlab/files/BernsteinD Module.pdf

5. Berthelot, P.: $D$-modules arithmétiques. I. Opérateurs différentiels de niveau fini. Ann. Sci. École Norm. Sup. (4) 29(2), 185-272 (1996)

6. Blickle, M., Mustaţă, M., Smith, K.E.: Discreteness and rationality of $F$-thresholds. Michigan Math. J. 57, 43-61 (2008). Special volume in honor of Melvin Hochster

7. Blickle, M., Mustaţă, M., Smith, K.E.: F-thresholds of hypersurfaces. Trans. Am. Math. Soc. 361(12), 6549-6565 (2009)

8. Ein, L., Lazarsfeld, R., Smith, K.E., Varolin, D.: Jumping coefficients of multiplier ideals. Duke Math. J. 123(3), 469-506 (2004) 
9. Emerton, M., Kisin, M.: The Riemann-Hilbert correspondence for unit $F$-crystals. Astérisque 293, vi+257 (2004)

10. Granville, A.: Arithmetic properties of binomial coefficients. I. Binomial coefficients modulo prime powers. In: Organic Mathematics (Burnaby, BC, 1995), vol. 20 of CMS Conf. Proc., pp. 253-276. Amer. Math. Soc., Providence, RI (1997)

11. Grothendieck, A.: Éléments de géométrie algébrique. IV. Étude locale des schémas et des morphismes de schémas IV. Inst. Hautes Études Sci. Publ. Math. 32, 361 (1967)

12. Hara, N., Yoshida, K.-I.: A generalization of tight closure and multiplier ideals. Trans. Am. Math. Soc. 355(8), 3143-3174 (2003). (electronic)

13. Kashiwara, M.: $B$-functions and holonomic systems. Rationality of roots of $B$-functions. Invent. Math. 38(1):33-53 (1976/77)

14. Kashiwara, M.: D-modules and microlocal calculus, vol. 217 of translations of mathematical monographs. American Mathematical Society, Providence, RI, 2003. Translated from the 2000 Japanese original by Mutsumi Saito, Iwanami Series in Modern Mathematics

15. Kollár, J.: Singularities of pairs. In: Algebraic Geometry-Santa Cruz 1995, vol. 62 of Proc. Sympos. Pure Math., pp. 221-287. Amer. Math. Soc., Providence, RI (1997)

16. Lazard, M.: Groupes analytiques p-adiques. Inst. Hautes Études Sci. Publ. Math. 26, 389-603 (1965)

17. Leykin, A., Walther, U.: Survey on the $d$-module $f^{s}$. arXiv preprint arXiv:1504.07516 (2015)

18. Lyubeznik, G.: $F$-modules: applications to local cohomology and $D$-modules in characteristic $p>0$. J. Reine Angew. Math. 491, 65-130 (1997)

19. Malgrange, B.: Polynômes de Bernstein-Sato et cohomologie évanescente. In: Analysis and Topology on Singular Spaces, II, III (Luminy, 1981), vol. 101 of Astérisque, pp. 243-267. Soc. Math. France, Paris (1983)

20. Mustaţă, M.: Bernstein-Sato polynomials in positive characteristic. J. Algebra 321(1), 128-151 (2009)

21. Mustaţă, M., Takagi, S., Watanabe. K.: F-thresholds and Bernstein-Sato polynomials. In: European Congress of Mathematics, pp 341-364. Eur. Math. Soc., Zürich (2005)

22. Saito, M.: Introduction to a theory of $b$-functions. arXiv preprint arXiv:math/0610783 (2006)

23. Smith, S.P.: The global homological dimension of the ring of differential operators on a nonsingular variety over a field of positive characteristic. J. Algebra 107(1), 98-105 (1987) 